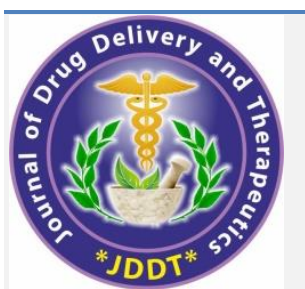

Open Access Full Text Article

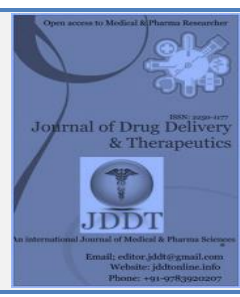

Review Article

\title{
Systematic Review on the Effectiveness of Strategies for Increasing Insulin Bioavailability in Oral Route Delivery Systems Based on Manufacturing Techniques and Materials Used
}

\author{
Krisna Adiva Puja (D), Ningrum Hendri Wahyu (D), Fimannuha Tamara Laily (D), Puspita Oktavia Eka*(D) \\ Department of Pharmacy, Faculty of Medicine, Brawijaya University, Malang 65145, East Java, Indonesia
}

Article Info:

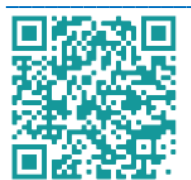

\section{Article History:}

Received 19 September 2021 Reviewed 20 October 2021 Accepted 24 October 2021 Published 15 November 2021

\section{Cite this article as:}

Krisna AP, Ningrum HW, Fimannuha TL, Puspita OE, Systematic Review on the Effectiveness of Strategies for Increasing Insulin Bioavailability in Oral Route Delivery Systems Based on Manufacturing Techniques and Materials Used Journal of Drug Delivery and Therapeutics. 2021 11(6):194-208

DOI: http://dx.doi.org/10.22270/jddt.v11i6.5132

*Address for Correspondence:

Puspita Oktavia Eka, Department of Pharmacy, Faculty of Medicine, Brawijaya University, Malang 65145, East Java, Indonesia

ORCID ID: https://orcid.org/0000-0001-6107-1089

\section{Abstract}

Diabetes is a metabolic disease characterized by hyperglycemia due to impaired insulin secretion, insulin action, or both. All patients with type 1 diabetes and many type 2 diabetes require insulin therapy to achieve reasonable glycemic control. During this time, insulin is given through the subcutaneous injection route because it can be destroyed by gastric acid when given orally. Until now, many studies have developed oral insulin therapy using various delivery system strategies. This systematic literature review aims to answer several questions about the effect of technique and material on increasing oral insulin bioavailability and the best technique and type of material that can produce the best oral insulin bioavailability. We searched for published articles regarding the development of oral route insulin. Bioavailability parameters were assessed based on plasma insulin levels for relative bioavailability values and/or plasma glucose levels for pharmacological bioavailability values. Conclusion: The manufacturing technique in the delivery system affects insulin stability in maintaining its conformation to provide a therapeutic effect. The type of substance affects insulin bioavailability through its properties in paving the way for insulin across various barriers in the digestive tract. To date, the best results in the development of oral insulin have obtained oral insulin bioavailability of $73.10 \%$ achieved by mesoporous silica nanoparticles (MSN) delivery system with layer-by-layer technique coated with [poly (methacrylic acid-co-vinyl triethoxylsilane)] (PMV)].

Keywords: bioavailability, diabetes, insulin, nanoparticles, oral delivery system.

\section{INTRODUCTION}

Diabetes is a non-communicable disease characterized by hyperglycemia due to impaired insulin secretion, insulin action, or both. Chronic hyperglycemia in diabetes can lead to failure of various organs, especially the eyes, kidneys, nerves, heart, and blood vessels $\mathbf{1}$. Globally, people with diabetes in 2019 are estimated at 9.3\% (463 million people), increasing to $10.2 \%$ (578 million) in 2030 and $10.9 \%$ (700 million) in $2045^{2}$. Meanwhile, in Indonesia, based on Basic Health Research data, people with diabetes increased from $6.9 \%$ in 2013 to $8.5 \%$ in 2018 , so the estimated number of sufferers in Indonesia reaches more than 16 million people (Kemenkes RI, 2018)2(a). All patients with type 1 diabetes and many patients with type 2 diabetes require insulin therapy to achieve reasonable glycemic control 3 .

Insulin is the most effective way to lower blood glucose, allowing the body to maintain glucose within a normal range 4. Insulin is given by subcutaneous injection because it can be destroyed by stomach acid if given orally 3 . However, daily insulin injections are considered ineffective because they cause pain at the injection site, are inconvenient, uncomfortable, and lead to low patient compliance $\mathbf{5}$. Therefore, many researchers have developed insulin administration via a convenient, non-invasive route such as the oral route. It is recognized as the most convenient and commonly used method of drug administration due to its ease of administration, high patient compliance, costeffectiveness, minimum sterility constraints, and flexible dosage form design. However, low bioavailability is a significant challenge in designing oral dosage forms ${ }^{6}$.

The bioavailability of a drug is the portion of the administered dose that reaches the systemic circulation 7 . Oral bioavailability is influenced by several factors such as water solubility, drug permeability, dissolution rate, firstpass metabolism, pre-systemic metabolism, and susceptibility to efflux mechanisms ${ }^{6}$. A drug must reach the desired drug concentration in the systemic circulation ${ }^{8}$. Insulin has low oral bioavailability due to the degradation of proteolytic enzymes and lack of intrinsic permeability through the intestinal epithelium ${ }^{9}$.

Several strategies have been carried out to develop insulin delivery systems via the oral route. Several reviews of 
articles related to the development of the oral route of insulin have also been carried out. A review of articles conducted by Singh et al. (2019) reported that oral route insulin bioavailability could be increased through encapsulation to insulin 10. In addition, Wong et al. (2021) reviewed articles on the characteristics of oral insulin preparation techniques and found that insulin bioavailability can be increased through various insulin preparation techniques used 11. However, there has been no review of articles that analyze the best materials and techniques in the manufacture of insulin that can increase the bioavailability of oral insulin, so this is the background of this study. This study conducted a Systematic Literature Review to determine whether the manufacturing technique and material used to develop oral route insulin therapy can affect insulin bioavailability. The review results are expected to provide information about the best techniques and types of ingredients in increasing the bioavailability of oral insulin. In the future, the results of this study can be helpful to facilitate the development of oral insulin with the best results and quality. To achieve the research objectives, the results of this Systematic Literature Review must be able to answer the following research questions (RQ):

1. Can the manufacturing technique and the type of material used in the delivery system affect the bioavailability of oral insulin?

2. What are the best manufacturing techniques and types of ingredients to increase the bioavailability of oral insulin?

To answer the formulation of these questions requires relevant research results. Therefore, in this Systematic Literature Review, limitations are given to the criteria of the article, namely:

1. Inclusion Criteria (IC 1): Articles published in 2015-2021.

2. Inclusion Criteria (IC 2): The article on insulin research using the oral route is the original article.

3. Inclusion Criteria (IC 3): Articles written in English or Indonesian.

4. Inclusion Criteria (IC 4): Article on the results of an oral insulin study with parameters for measuring bioavailability (insulin levels in plasma and glucose levels in plasma) using in vivo research method.

5. Inclusion Criteria (IC 5): Insulin is formulated in the form of a nanomedicine delivery system (liposome, solid lipid nanoparticle, polymeric nanoparticle, inorganic nanoparticle, insulin emulsion, nanogels).

Thus, not all articles are used to answer this question. Articles that do not meet the data inclusion criteria or articles that contain data on insulin but have the following criteria will not be used to answer research questions. However, it is possible to use it as supporting data only. The article exclusion criteria are as follows:

1. Exclusion Criteria (EC 1): The article does not have copyright.

\section{METHODS}

The study was conducted based on the Systematic Literature Review method used by Rowley \& Slack (2004) and based on the Preferred Reporting Items for Systematic Review and Meta-Analyses (PRISMA) protocol 12,13 (Figure 1). The research was carried out in 5 stages, namely (a) scanning documents, (b) making documentation, (c) arranging Literature Review, (d) writing Literature Review, and (e) compiling a bibliography. Scanning documents is done by identifying keywords and articles that must be included in the Systematic Literature Review. Documentation is made by listing the references from which the articles were downloaded. Structuring the Literature Review is done by identifying the main themes and then sorting them to select articles. Writing literature according to the themes identified in the previous step was performed. The bibliography is compiled by including all sources referenced in the preparation of the Systematic Literature Review.

\section{a. Keyword Identification}

The article search begins with determining the combination of keywords that match the formulation of research questions to scan documents on a digital database. The combination of keywords used is insulin AND bioavailability AND "oral administration" OR "oral delivery system" AND liposome OR "solid lipid nanoparticle" OR "polymeric nanoparticle" OR "inorganic nanoparticle" OR "insulin emulsion" OR nanogels.

\section{b. Article Search}

Based on article searches that have been carried out through three digital databases, 1000 articles were obtained from Google Scholar, seven articles from Pubmed, and 800 articles from Crossref. The total number of articles obtained is 1807 article titles. The first stage is metadata or combining articles that have been obtained from the three sources in .xlsx format. The next step is initial screening, sorting the early stages of articles with the same title and publisher (duplicate screening). Duplicated articles from 1807 articles are 97 articles. The total number of the article after the duplication was removed was 1710 articles. Based on the screening results through the title and abstract of the article against the suitability of the inclusion and exclusion criteria, it was found that the number of articles that met the inclusion criteria was 77 articles. The results of the article assessment using the Checklist for Quasi-Experimental Studies (NonRandomized Experimental Studies) from the Joanna Briggs Institute obtained articles that meet the assessment or are eligible are 76 articles.

\section{c. Article Profile Description}

A description of the article profile was carried out on the selected articles, including article title, author's name, year of publication, publisher, citation per year, manufacturing technique, type of delivery system, formula content, test method, bioavailability test parameters, as well as the resulting bioavailability to be extracted and stored in a format. xlsx. Figure 2 shows the trend of article publication from year to year. 


\section{Study identification via database}

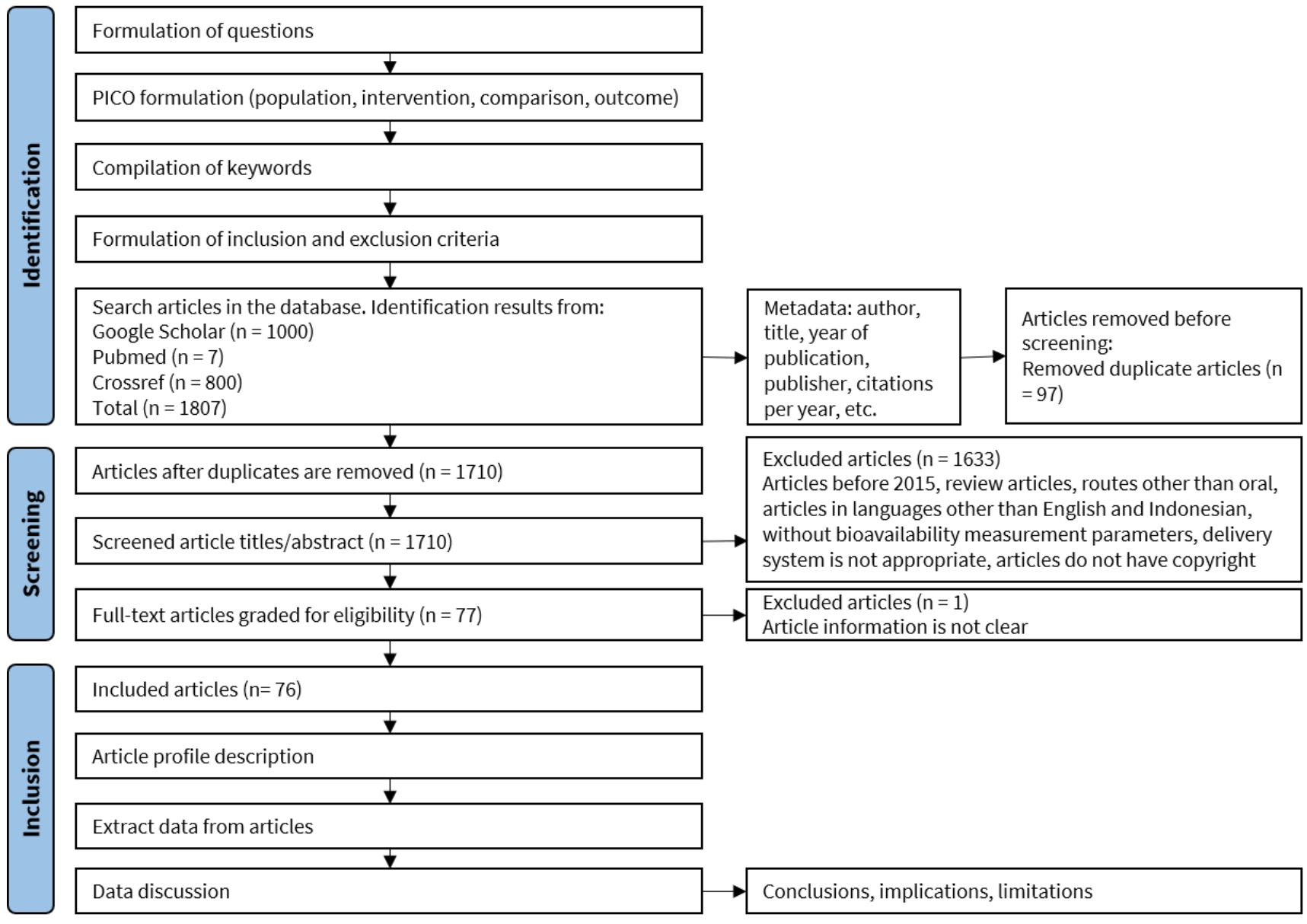

Figure 1: Systematic Literature Review Process

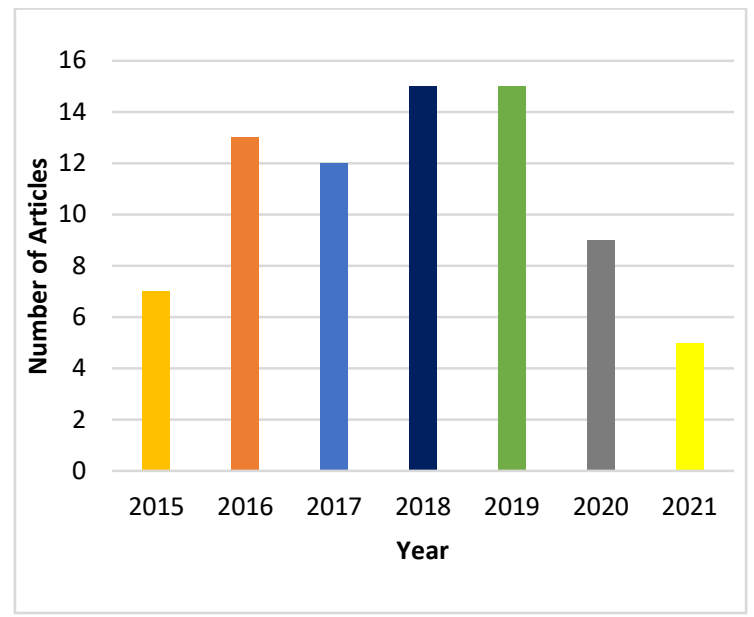

Figure 2: Trend of the Number of Articles Each Year in the 2015-2021 Range

The types of delivery systems used vary, including polymeric nanoparticles and lipid nanoparticles. Each country's contribution to the publication of research on the development of oral insulin is shown in Figure 3. Countries were determined based on the authors' affiliation-the country with the highest number of studies in China, followed by India in the second position.

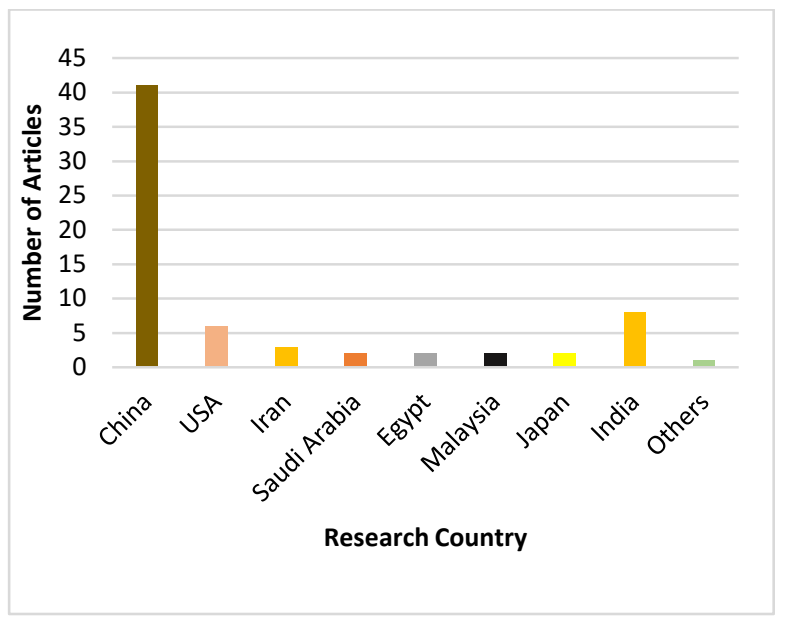

Figure 3: Country Profile of Research Site

All articles have been published in journals with Impact Factors showing that the journal is of high quality and contributes to the research field. Citation analysis is also used to determine the relevance between articles and how many articles are quoted. The citation of an article also shows that the article is of high quality. Table 1 shows the ranking of articles based on citation analysis. 
Table 1: Articles by Number of Citations

\begin{tabular}{|c|c|c|c|c|c|c|c|c|c|c|c|}
\hline Rank & Author & Year & $\begin{array}{l}\text { Citation } \\
\text { per Year }\end{array}$ & Rank & Author & Year & $\begin{array}{l}\text { Citation } \\
\text { per Year }\end{array}$ & Rank & Author & Year & $\begin{array}{l}\text { Citation } \\
\text { per Year }\end{array}$ \\
\hline 1 & He et al (20) & 2017 & 33,5 & 27 & $\begin{array}{l}\text { Malathi et } \\
\text { al (21) }\end{array}$ & 2015 & 8,83 & 53 & $\begin{array}{l}\text { Wu et al } \\
(22)\end{array}$ & 2019 & 3,5 \\
\hline 2 & Liu et al (23) & 2016 & 33,2 & 28 & $\begin{array}{l}\text { Wu et al } \\
(24)\end{array}$ & 2017 & 8,75 & 54 & $\begin{array}{l}\text { Alsulays et } \\
\text { al (25) }\end{array}$ & 2019 & 3,5 \\
\hline 3 & $\begin{array}{l}\text { Wang et al } \\
(26)\end{array}$ & 2019 & 32 & 29 & Ji et al (27) & 2019 & 8,5 & 55 & $\begin{array}{l}\text { Agrawal et } \\
\text { al (28) }\end{array}$ & 2015 & 3,5 \\
\hline 4 & Fan et al (29) & 2018 & 30 & 30 & $\begin{array}{l}\text { Omid et al } \\
(30)\end{array}$ & 2017 & 8 & 56 & $\begin{array}{l}\text { Zhang et al } \\
\text { (31) }\end{array}$ & 2017 & 3,25 \\
\hline 5 & $\begin{array}{l}\text { Mumuni et al } \\
\text { (32) }\end{array}$ & 2020 & 25 & 31 & $\begin{array}{l}\text { Ukai et al } \\
\text { (33) }\end{array}$ & 2020 & 8 & 57 & $\begin{array}{l}\text { Fang et al } \\
(34)\end{array}$ & 2018 & 3 \\
\hline 6 & $\begin{array}{l}\text { Han et al } \\
(14)\end{array}$ & 2020 & 21 & 32 & $\begin{array}{l}\text { Zhang et al } \\
\text { (35) }\end{array}$ & 2015 & 7,83 & 58 & $\begin{array}{l}\text { Sun et al } \\
(36)\end{array}$ & 2019 & 3 \\
\hline 7 & Li et al (37) & 2017 & 19,5 & 33 & $\begin{array}{l}\text { Liu et al } \\
\text { (38) }\end{array}$ & 2016 & 6,6 & 59 & $\begin{array}{l}\text { Sahoo et al } \\
\text { (39) }\end{array}$ & 2019 & 3 \\
\hline 8 & $\begin{array}{l}\text { Sheng et al } \\
(40)\end{array}$ & 2016 & 19,4 & 34 & $\begin{array}{l}\text { Sun et al } \\
(41)\end{array}$ & 2015 & 6,33 & 60 & $\begin{array}{l}\text { Alfaro et al } \\
(42)\end{array}$ & 2020 & 3 \\
\hline 9 & $\begin{array}{l}\text { Sheng et al } \\
\text { (43) }\end{array}$ & 2015 & 19,17 & 35 & $\begin{array}{l}\text { Guha et al } \\
\text { (19) }\end{array}$ & 2016 & 6,2 & 61 & $\begin{array}{l}\text { Wang et al } \\
(44)\end{array}$ & 2018 & 2,67 \\
\hline 10 & Wu et al (45) & 2018 & 19 & 36 & $\begin{array}{l}\text { Yazdi et al } \\
(46)\end{array}$ & 2020 & 6 & 62 & $\begin{array}{l}\text { Hu et al } \\
(47)\end{array}$ & 2019 & 2,5 \\
\hline 11 & Liu et al (48) & 2019 & 18 & 37 & $\begin{array}{l}\text { Liu et al } \\
(49)\end{array}$ & 2019 & 6 & 63 & $\begin{array}{l}\text { Boushra et } \\
\text { al (50) }\end{array}$ & 2019 & 2,5 \\
\hline 12 & $\begin{array}{l}\text { Shan et al } \\
\text { (51) }\end{array}$ & 2016 & 17,2 & 38 & Ji et al (52) & 2017 & 5,75 & 64 & $\begin{array}{l}\text { Xie et al } \\
(53)\end{array}$ & 2018 & 2,33 \\
\hline 13 & $\begin{array}{l}\text { Tian et al } \\
(54)\end{array}$ & 2018 & 16 & 39 & $\begin{array}{l}\text { Guo et al } \\
\text { (55) }\end{array}$ & 2016 & 5,4 & 65 & $\begin{array}{l}\text { Zhang et al } \\
\text { (56) }\end{array}$ & 2021 & 2 \\
\hline 14 & $\begin{array}{l}\text { Alibolandi et } \\
\text { al (57) }\end{array}$ & 2016 & 15,4 & 40 & $\begin{array}{l}\text { Kim et al } \\
(58)\end{array}$ & 2018 & 5,33 & 66 & $\begin{array}{l}\text { Zhang et al } \\
\text { (59) }\end{array}$ & 2018 & 1,67 \\
\hline 15 & $\begin{array}{l}\text { Verma et al } \\
(60)\end{array}$ & 2015 & 15 & 41 & $\begin{array}{l}\text { Fukuoka et } \\
\text { al (61) }\end{array}$ & 2018 & 5,33 & 67 & $\begin{array}{l}\text { Yan et al } \\
(62)\end{array}$ & 2019 & 1,5 \\
\hline 16 & $\begin{array}{l}\text { Wang et al } \\
(63)\end{array}$ & 2017 & 15 & 42 & $\begin{array}{l}\text { Chen et al } \\
(64)\end{array}$ & 2019 & 5 & 68 & $\begin{array}{l}\text { Bahman et } \\
\text { al (65) }\end{array}$ & 2020 & 1 \\
\hline 17 & $\begin{array}{l}\text { Shrestha et } \\
\text { al (66) }\end{array}$ & 2016 & 15 & 43 & $\begin{array}{l}\text { Zhou et al } \\
\text { (67) }\end{array}$ & 2020 & 5 & 69 & $\begin{array}{l}\text { Winarti et } \\
\text { al (68) }\end{array}$ & 2018 & 0,67 \\
\hline 18 & Liu et al (18) & 2016 & 14 & 44 & $\begin{array}{l}\text { Sun et al } \\
(69)\end{array}$ & 2016 & 4,5 & 70 & $\begin{array}{l}\text { Zhang et al } \\
\text { (70) }\end{array}$ & 2021 & 0 \\
\hline 19 & $\begin{array}{l}\text { Zeng et al } \\
\text { (71) }\end{array}$ & 2018 & 11 & 45 & $\begin{array}{l}\text { He et al } \\
(72)\end{array}$ & 2015 & 4,5 & 71 & $\begin{array}{l}\text { Ansari et al } \\
\text { (73) }\end{array}$ & 2016 & 0 \\
\hline 20 & Niu et al (74) & 2017 & 11 & 46 & $\begin{array}{l}\text { Boushra et } \\
\text { al (75) }\end{array}$ & 2016 & 4,4 & 72 & $\begin{array}{l}\text { Koland et } \\
\text { al (76) }\end{array}$ & 2021 & 0 \\
\hline 21 & $\begin{array}{l}\text { Deng et al } \\
\text { (77) }\end{array}$ & 2017 & 10 & 47 & $\begin{array}{l}\text { Singh et al } \\
\text { (78) }\end{array}$ & 2018 & 4,33 & 73 & $\begin{array}{l}\text { Heade et al } \\
\text { (79) }\end{array}$ & 2021 & 0 \\
\hline 22 & $\begin{array}{l}\text { Chen et al } \\
(80)\end{array}$ & 2017 & 10 & 48 & $\begin{array}{l}\text { Agrawal et } \\
\text { al (81) }\end{array}$ & 2017 & 4,25 & 74 & $\begin{array}{l}\text { Kaur et al } \\
(82)\end{array}$ & 2021 & 0 \\
\hline 23 & Zhu et al (83) & 2016 & 10 & 49 & $\begin{array}{l}\text { Urimi et al } \\
(84)\end{array}$ & 2019 & 4 & 75 & $\begin{array}{l}\text { Wang et al } \\
(85)\end{array}$ & 2020 & 0 \\
\hline 24 & Xu et al (86) & 2017 & 9,25 & 50 & $\begin{array}{l}\text { El-Leithy et } \\
\text { al (87) }\end{array}$ & 2019 & 4 & 76 & $\begin{array}{l}\text { Elkhatib et } \\
\text { al (88) }\end{array}$ & 2021 & 0 \\
\hline 25 & $\begin{array}{l}\text { Chen et al } \\
\text { (17) }\end{array}$ & 2019 & 9 & 51 & $\begin{array}{l}\text { Elsayed et } \\
\text { al (89) }\end{array}$ & 2018 & 4 & & & & \\
\hline 26 & $\begin{array}{l}\text { Zheng et al } \\
(90)\end{array}$ & 2018 & 9 & 52 & $\begin{array}{l}\text { Jaafar \& } \\
\text { Hamid (15) }\end{array}$ & 2019 & 3,5 & & & & \\
\hline
\end{tabular}




\section{RESULTS AND DISCUSSIONS}

The complete summary results of the 76 selected articles can be seen in Supplementary File 1. Selected articles are classified based on the research questions that will be answered in this study, namely (1) the effect of the technique on increasing oral insulin bioavailability (RQ1), (2) the best manufacturing technique in increasing the bioavailability of oral insulin (RQ2), (3) the effect of the type of substance on increasing the bioavailability of oral insulin (RQ3), and (4) the best type of substance in increasing the bioavailability of oral insulin (RQ4).

\section{a. Oral Insulin Development Challenges}

Insulin is a high-molecular-weight protein that is highly hydrophilic, so it cannot cross the digestive tract properly due to many barriers. In order to work orally, insulin must pass through three main physiological barriers, namely 1) insulin must be able to withstand a very acidic gastric $\mathrm{pH}$ $(\mathrm{pH}=1-3)$ and proteolytic enzymes that can degrade/denature insulin; 2) insulin must be able to penetrate the mucous layer that protects the intestinal epithelial surface, and 3) insulin must be able to pass through the intestinal epithelial cell layer to enter the systemic circulation $\mathbf{1 4}$. Therefore, insulin delivery systems must be designed effectively to protect insulin from obstacles in the digestive tract, biocompatible to maintain the conformational integrity of insulin to remain pharmacologically active, and able to load more insulin in the accurate concentrations that it can control insulin levels for optimal blood glucose $\mathbf{1 5}$.

\section{b. Effect of Technique on Increasing Oral Insulin Bioavailability}

The manufacturing technique is a factor that plays a role in efforts to increase the bioavailability of oral insulin. The manufacturing technique must pay attention to the physicochemical properties of insulin in order to maintain insulin stability until the end. Based on the review results, the technique used to manufacture an oral insulin delivery system can be seen in Table 2 . The most widely used technique is double emulsion solvent evaporation, which is $18.42 \%$. The hydrophilicity and hydrophobicity of the active substance are very important to determine the manufacturing technique used in the delivery system. The double emulsion technique [water in oil in water (w/o/w)], also known as emulsion, is a complex system in which the dispersed phase droplets consist of small dispersed phases. This technique is widely used to encapsulate proteins because it can protect against degradation due to acidic gastric $\mathrm{pH}$ and proteolytic enzymes in the small intestine. In addition, this technique can help drugs achieve sustained release, are biocompatible and biodegradable, and can encapsulate two types of hydrophilic and hydrophobic drugs separately and simultaneously. However, this technique has several disadvantages, including the need for high shear stresses and high-pressure homogenization so that the protein tends to denature and form aggregates due to the high shear force and the significant interface exposure between the aqueous and the organic phase. In addition, the resulting particles are relatively heterogeneous, the particle size is sensitive to various parameters of the manufacturing process, and this technique has not had excellent encapsulation efficiency ${ }^{16}$. Like the research that has been done by Chen et al. (2019) resulted in an oral insulin bioavailability of $7.51 \%$, which is still relatively low 17 .

\section{c. Effect of Type of Substance on Increasing Oral Insulin Bioavailability}

Several ingredients have been reported to increase oral insulin bioavailability, which can be seen in Table 2 . Chitosan is the most widely used material in oral insulin delivery systems because it has nontoxic and biocompatible properties, can mediate the opening of tight junctions between epithelial cells reversibly, and can increase permeability via the paracellular pathway. The opening of tight junctions by chitosan is caused by the interaction between chitosan and integrin receptors on the cell membrane, which causes the conformation of integrin receptors which can then damage the tight junction area. The use of chitosan can also prevent nanoparticles from complicated intracellular transport and prevent the enzymatic degradation of insulin in lysosomes ${ }^{\mathbf{1 8}}$. Research conducted by Jafar \& Hamid (2019) showed that the use of chitosan polymer could increase the bioavailability of oral insulin by $40.23 \% 15$.

\section{d. The Best Techniques and Types of Materials in Increasing Oral Insulin Bioavailability}

Based on the results of systematic studies that have been carried out, there are the best techniques and materials based on bioavailability parameters that can be recommended in the development of oral insulin routes. These findings are based on research by Guha et al. (2016) using a mesoporous silica nanoparticles (MSN) delivery system with a layer-by-layer technique coated with a polymer [poly (methacrylic acid-co-vinyl triethoxylsilane)] (PMV). PMV was obtained from the synthesis of methacrylic acid (MAA) and vinyl triethoxylsilane (VTES). PMV polymers are sensitive to $\mathrm{pH}$ values so that their release can be targeted in the intestine with the prolonged-release for 6 hours. PMV can protect insulin from the degradation of proteolytic enzymes and gastric acid environment. Encapsulation of insulin with PMV can help insulin cross the intestinal mucosa through paracellular and transcellular transport, then quickly absorbed by intestinal epithelial cells and directly reach the systemic circulation. The layer-bylayer technique is reported to produce a large nanoparticle surface area of $304.3921 \mathrm{~m} 2 / \mathrm{g}$ and an adsorption pore width with a smaller dimension of $64.7844 \mathrm{~nm}$ to increase insulin absorption to obtain a significantly increased bioavailability of $73.10 \%{ }^{19}$. 
Table 2: Comprehensive Summary of Oral Insulin Development Articles

\begin{tabular}{|c|c|c|c|c|c|c|c|c|c|c|}
\hline $\begin{array}{l}\mathrm{N} \\
\mathrm{o}\end{array}$ & Author & $\begin{array}{l}\text { Yea } \\
\mathrm{r}\end{array}$ & Publisher & $\begin{array}{l}\text { Journal and } \\
\text { Impact Factor }\end{array}$ & Method & $\begin{array}{l}\text { Delivery } \\
\text { System }\end{array}$ & Formulation & $\begin{array}{l}\text { Test } \\
\text { Meth } \\
\text { od }\end{array}$ & $\begin{array}{l}\text { BA } \\
\text { Parameters }\end{array}$ & $\begin{array}{l}\text { Theme } \\
\text { Classifica } \\
\text { tion }\end{array}$ \\
\hline 1 & Ji et al & $\begin{array}{l}20 \\
19\end{array}$ & Elsevier & $\begin{array}{l}\text { Journal of } \\
\text { Controlled } \\
\text { Release } \\
(7,633)\end{array}$ & $\begin{array}{l}\text { Antisolvent } \\
\text { coprecipitati } \\
\text { on }\end{array}$ & $\begin{array}{l}\text { nanocompo } \\
\text { cite }\end{array}$ & $\begin{array}{l}\text { Carboxymethylated } \\
\text { short-chain amylose; } \\
\text { zein; chitosan. }\end{array}$ & in & $\begin{array}{l}\text { plasma } \\
\text { insulin level }\end{array}$ & RQ3, RQ4 \\
\hline 2 & $\begin{array}{l}\text { Kim et } \\
\text { al }\end{array}$ & $\begin{array}{l}20 \\
18\end{array}$ & $\begin{array}{l}\text { ACS } \\
\text { Publicatio } \\
\text { ns }\end{array}$ & $\begin{array}{l}\text { Molecular } \\
\text { Pharmaceutic } \\
\text { s }(4,44)\end{array}$ & $\mathrm{N} / \mathrm{A}$ & liposome & $\begin{array}{l}\text { Chondroitin sulfate-g- } \\
\text { taurocholic acid (CST). }\end{array}$ & $\begin{array}{l}\text { in } \\
\text { vivo }\end{array}$ & $\begin{array}{l}\text { plasma } \\
\text { insulin level }\end{array}$ & RQ3, RQ4 \\
\hline 3 & $\begin{array}{l}\text { Yazdi } \\
\text { et al }\end{array}$ & $\begin{array}{l}20 \\
20\end{array}$ & Elsevier & $\begin{array}{l}\text { Colloids and } \\
\text { Surfaces B: } \\
\text { Biointerfaces } \\
(5,268)\end{array}$ & $\begin{array}{l}\text { thin film } \\
\text { hydration }\end{array}$ & liposome & $\begin{array}{l}\text { PEG, folic acid, } \\
\text { hydrogenated soya } \\
\text { phosphatidylcholine } \\
\text { (HSPC). }\end{array}$ & 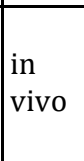 & $\begin{array}{l}\text { plasma } \\
\text { insulin level }\end{array}$ & $\begin{array}{l}\text { RQ1, } \\
\text { RQ2, } \\
\text { RQ3, RQ4 }\end{array}$ \\
\hline 4 & $\begin{array}{l}\text { Wang } \\
\text { et al }\end{array}$ & $\begin{array}{l}20 \\
18\end{array}$ & $\begin{array}{l}\text { Taylor \& } \\
\text { Francis }\end{array}$ & $\begin{array}{l}\text { Pharmaceutic } \\
\text { al } \\
\text { Development } \\
\text { and } \\
\text { Technology } \\
(2,347)\end{array}$ & $\begin{array}{l}\text { emulsion } \\
\text { polymerizati } \\
\text { on }\end{array}$ & nanogel & $\begin{array}{l}\text { Hydroxyethyl } \\
\text { methacrylate (HEMA). }\end{array}$ & $\begin{array}{l}\text { in } \\
\text { vivo }\end{array}$ & $\begin{array}{l}\text { plasma } \\
\text { insulin level, } \\
\text { plasma } \\
\text { glucose } \\
\text { level }\end{array}$ & $\begin{array}{l}\text { RQ1, } \\
\text { RQ2, } \\
\text { RQ3, RQ4 }\end{array}$ \\
\hline 5 & $\begin{array}{l}\text { Agraw } \\
\text { al et al }\end{array}$ & $\begin{array}{l}20 \\
17\end{array}$ & $\begin{array}{l}\text { ACS } \\
\text { Publicatio } \\
\text { ns }\end{array}$ & $\begin{array}{l}\text { Molecular } \\
\text { Pharmaceutic } \\
\text { s }(4,44)\end{array}$ & hydrotope & nanoparticl & lesitin, pluronic $\mathrm{f}-127$ & $\begin{array}{l}\text { in } \\
\text { vivo }\end{array}$ & $\begin{array}{l}\text { plasma } \\
\text { glucose } \\
\text { level }\end{array}$ & $\begin{array}{l}\text { RQ1, } \\
\text { RQ2, } \\
\text { RQ3, RQ4 }\end{array}$ \\
\hline 6 & $\begin{array}{l}\text { Wang } \\
\text { et al }\end{array}$ & $\begin{array}{l}20 \\
19\end{array}$ & $\begin{array}{l}\text { Wiley } \\
\text { Online } \\
\text { Library }\end{array}$ & $\begin{array}{l}\text { Advanced } \\
\text { Healthcare } \\
\text { Materials } \\
(7,367)\end{array}$ & $\begin{array}{l}\text { thin film } \\
\text { hydration }\end{array}$ & liposome & $\begin{array}{l}\text { EPC, cholesterol, } \\
\text { DOTAP }\end{array}$ & $\begin{array}{l}\text { in } \\
\text { vivo }\end{array}$ & $\begin{array}{l}\text { plasma } \\
\text { insulin level }\end{array}$ & $\begin{array}{l}\text { RQ1, } \\
\text { RQ2, } \\
\text { RQ3, RQ4 }\end{array}$ \\
\hline 7 & $\begin{array}{l}\text { Singh } \\
\text { et al }\end{array}$ & $\begin{array}{l}20 \\
18\end{array}$ & $\begin{array}{l}\text { Future } \\
\text { Medicine }\end{array}$ & $\begin{array}{l}\text { Nanomedicin } \\
\text { e (London) } \\
(4,727)\end{array}$ & $\mathrm{N} / \mathrm{A}$ & nanoparticl & $\begin{array}{l}\text { Pluronic F-127, GMO, } \\
\text { propylene glycol. }\end{array}$ & $\begin{array}{l}\text { in } \\
\text { vivo }\end{array}$ & $\begin{array}{l}\text { plasma } \\
\text { insulin level, } \\
\text { plasma } \\
\text { glucose } \\
\text { level }\end{array}$ & RQ3. RQ4 \\
\hline 8 & $\begin{array}{l}\text { Jaafar } \\
\& \\
\text { Hamid }\end{array}$ & $\begin{array}{l}20 \\
19\end{array}$ & $\begin{array}{l}\text { Ingenta } \\
\text { Connect }\end{array}$ & $\begin{array}{l}\text { Current Drug } \\
\text { Delivery } \\
(1,582)\end{array}$ & $\begin{array}{l}\text { polyelectroly } \\
\text { te } \\
\text { complexatio } \\
\mathrm{n} \text { and } \\
\text { ionotropic } \\
\text { gelation }\end{array}$ & $\begin{array}{l}\text { nanoparticl } \\
\text { e }\end{array}$ & $\begin{array}{l}\text { Alginate, calcium } \\
\text { chloride }(\mathrm{CaCl} 2), \\
\text { Pluronic-68, dextran } \\
\text { sulfate, chitosan }\end{array}$ & in & $\begin{array}{l}\text { plasma } \\
\text { insulin level }\end{array}$ & $\begin{array}{l}\text { RQ1, } \\
\text { RQ2, } \\
\text { RQ3, RQ4 }\end{array}$ \\
\hline 9 & $\begin{array}{l}\text { Zhang } \\
\text { et al }\end{array}$ & $\begin{array}{l}20 \\
20\end{array}$ & $\begin{array}{l}\text { Royal } \\
\text { Society of } \\
\text { Chemistry }\end{array}$ & $\begin{array}{l}\text { Nanoscale } \\
(6,895)\end{array}$ & $\begin{array}{l}\text { thin film } \\
\text { rehydration }\end{array}$ & $\begin{array}{l}\text { nanoliposo } \\
\text { me }\end{array}$ & $\begin{array}{l}\text { Hydrogenated soybean } \\
\text { phosphatidylcholine } \\
\text { (HSPC), and 1,2- } \\
\text { dipalmitoyl-sn-glycero- } \\
\text { 3-phosphoglycerol, } \\
\text { sodium salt (DPPG) }\end{array}$ & $\begin{array}{l}\text { in } \\
\text { vivo }\end{array}$ & $\begin{array}{l}\text { plasma } \\
\text { insulin level }\end{array}$ & $\begin{array}{l}\text { RQ1, } \\
\text { RQ2, } \\
\text { RQ3, RQ4 }\end{array}$ \\
\hline $\begin{array}{l}1 \\
0\end{array}$ & $\begin{array}{l}\text { Deng } \\
\text { et al }\end{array}$ & $\begin{array}{l}20 \\
17\end{array}$ & Elsevier & \begin{tabular}{|l|} 
Nanomedicin \\
e \\
Nanotechnolo \\
gy Biology \\
Medicine \\
$(6,458)$
\end{tabular} & $\begin{array}{l}\text { ionic cross- } \\
\text { linking/in } \\
\text { situ } \\
\text { reduction }\end{array}$ & nanoparticl & $\begin{array}{l}\text { Chitosan, Reduced L- } \\
\text { glutathione (GSH), } \\
\text { sodium selenite } \\
\text { (Na2SeO3). }\end{array}$ & in & $\begin{array}{l}\text { plasma } \\
\text { insulin level }\end{array}$ & $\begin{array}{l}\text { RQ1, } \\
\text { RQ2, } \\
\text { RQ3, RQ4 }\end{array}$ \\
\hline $\begin{array}{l}1 \\
1\end{array}$ & $\begin{array}{l}\text { Wu et } \\
\text { al }\end{array}$ & $\begin{array}{l}20 \\
19\end{array}$ & Elsevier & $\begin{array}{l}\text { Journal of } \\
\text { Pharmaceutic } \\
\text { al Sciences } \\
(3,534)\end{array}$ & $\begin{array}{l}\text { reversed- } \\
\text { phase } \\
\text { evaporation }\end{array}$ & liposome & $\begin{array}{l}\text { Chitosan, deoxycholic } \\
\text { acid. }\end{array}$ & in & $\begin{array}{l}\text { plasma } \\
\text { insulin level }\end{array}$ & $\begin{array}{l}\text { RQ1, } \\
\text { RQ2, } \\
\text { RQ3, RQ4 }\end{array}$ \\
\hline $\begin{array}{l}1 \\
2\end{array}$ & $\begin{array}{l}\text { Liu et } \\
\text { al }\end{array}$ & $\begin{array}{l}20 \\
16\end{array}$ & $\begin{array}{l}\text { Dove } \\
\text { Medical } \\
\text { Press } \\
\text { Limited }\end{array}$ & $\begin{array}{l}\text { International } \\
\text { Journal of } \\
\text { Nanomedicin } \\
\text { e }(6,400)\end{array}$ & $\begin{array}{l}\text { modified } \\
\text { solvent- } \\
\text { injection }\end{array}$ & $\begin{array}{l}\text { nanoparticl } \\
\text { e }\end{array}$ & Lecithin, chitosan. & in & \begin{tabular}{|l|} 
glucose \\
insulin level \\
(pharmacol \\
ogical \\
bioavailabili \\
ty)
\end{tabular} & $\begin{array}{l}\text { RQ1, } \\
\text { RQ2, } \\
\text { RQ3, RQ4 }\end{array}$ \\
\hline
\end{tabular}




\begin{tabular}{|c|c|c|c|c|c|c|c|c|c|c|}
\hline $\begin{array}{l}\mathrm{N} \\
\mathrm{o}\end{array}$ & Author & $\begin{array}{l}\text { Yea } \\
\mathrm{r}\end{array}$ & Publisher & $\begin{array}{l}\text { Journal and } \\
\text { Impact Factor }\end{array}$ & Method & $\begin{array}{l}\text { Delivery } \\
\text { System }\end{array}$ & Formulation & $\begin{array}{l}\text { Test } \\
\text { Meth } \\
\text { od }\end{array}$ & $\begin{array}{l}\text { BA } \\
\text { Parameters }\end{array}$ & $\begin{array}{l}\text { Theme } \\
\text { Classifica } \\
\text { tion }\end{array}$ \\
\hline $\begin{array}{l}1 \\
3\end{array}$ & $\begin{array}{l}\text { Alibola } \\
\text { ndi et } \\
\text { al }\end{array}$ & $\begin{array}{l}20 \\
16\end{array}$ & Elsevier & $\begin{array}{l}\text { Journal of } \\
\text { Controlled } \\
\text { Release } \\
(7,633)\end{array}$ & $\begin{array}{l}\text { modified } \\
\text { direct } \\
\text { hydration }\end{array}$ & $\begin{array}{l}\text { polymerso } \\
\text { me }\end{array}$ & Dextran-PLGA. & in & $\begin{array}{l}\text { plasma } \\
\text { insulin level }\end{array}$ & $\begin{array}{l}\text { RQ1, } \\
\text { RQ2, } \\
\text { RQ3, RQ4 }\end{array}$ \\
\hline $\begin{array}{l}1 \\
4\end{array}$ & Ji et al & $\begin{array}{l}20 \\
17\end{array}$ & $\begin{array}{l}\text { ACS } \\
\text { Publicatio } \\
\text { ns }\end{array}$ & $\begin{array}{l}\text { Journal of } \\
\text { Agricultural } \\
\text { and Food } \\
\text { Chemistry } \\
(4,192)\end{array}$ & $\mathrm{N} / \mathrm{A}$ & $\begin{array}{l}\text { nanocompo } \\
\text { site }\end{array}$ & $\begin{array}{l}\text { short chain glucan } \\
\text { (SGC), } \\
\text { proanthocyanidins } \\
(\text { PAC). }\end{array}$ & in & \begin{tabular}{|l} 
glucose \\
insulin level
\end{tabular} & RQ3, RQ4 \\
\hline $\begin{array}{l}1 \\
5\end{array}$ & $\begin{array}{l}\text { Han et } \\
\text { al }\end{array}$ & $\begin{array}{l}20 \\
20\end{array}$ & Nature & \begin{tabular}{|l|} 
Nature \\
Nanotechnolo \\
gy $(33,407)$
\end{tabular} & $\mathrm{N} / \mathrm{A}$ & micelles & $\begin{array}{l}\text { polimer betaine } \\
\text { zwitterionic } \\
\text { (polycarboxybetaine, } \\
\text { PCB) terkonjugasi } \\
\text { menjadi 1,2 distearoyl- } \\
\text { sn-glycero-3- } \\
\text { phosphoethanolamine } \\
\text { (DSPE) }\end{array}$ & $\begin{array}{l}\text { in } \\
\text { vivo }\end{array}$ & \begin{tabular}{|l|} 
plasma \\
insulin level
\end{tabular} & RQ3, RQ4 \\
\hline $\begin{array}{l}1 \\
6\end{array}$ & $\begin{array}{l}\text { Chen } \\
\text { et al }\end{array}$ & $\begin{array}{l}20 \\
17\end{array}$ & Springer & $\begin{array}{l}\text { AAPS } \\
\text { PharmSciTec } \\
\text { h }(3,246)\end{array}$ & $\begin{array}{l}\text { ionotropic } \\
\text { gelation }\end{array}$ & nanoparticl & $\begin{array}{l}\text { Chitosan, eudragit } \\
\text { S100, transcriptional } \\
\text { peptide (tat) }\end{array}$ & $\begin{array}{l}\text { in } \\
\text { vivo }\end{array}$ & $\begin{array}{l}\text { plasma } \\
\text { insulin level }\end{array}$ & $\begin{array}{l}\text { RQ1, } \\
\text { RQ2, } \\
\text { RQ3, RQ4 }\end{array}$ \\
\hline $\begin{array}{l}1 \\
7\end{array}$ & $\begin{array}{l}\text { Urimi } \\
\text { et al }\end{array}$ & $\begin{array}{l}20 \\
19\end{array}$ & Springer & $\begin{array}{l}\text { AAPS } \\
\text { PharmSciTec } \\
\text { h }(3,246)\end{array}$ & $\begin{array}{l}\text { ionotropic } \\
\text { gelation }\end{array}$ & $\begin{array}{l}\text { nanoparticl } \\
\text { e }\end{array}$ & $\begin{array}{l}\text { Chitosan solution (1 } \\
\text { mg/mL), PSS } \\
\text { (poly(sodium 4- } \\
\text { styrenesulfonate)), } \\
\text { PGA }(\gamma \text {-polyglutamic } \\
\text { acid)] }\end{array}$ & $\begin{array}{l}\text { in } \\
\text { vivo }\end{array}$ & $\begin{array}{l}\text { glucose } \\
\text { insulin level }\end{array}$ & $\begin{array}{l}\text { RQ1, } \\
\text { RQ2, } \\
\text { RQ3, RQ4 }\end{array}$ \\
\hline $\begin{array}{l}1 \\
8\end{array}$ & $\begin{array}{l}\text { Chen } \\
\text { et al }\end{array}$ & $\begin{array}{l}20 \\
19\end{array}$ & $\begin{array}{l}\text { Taylor \& } \\
\text { Francis }\end{array}$ & $\begin{array}{l}\text { Journal of } \\
\text { Microencapsu } \\
\text { lation }(5,82)\end{array}$ & \begin{tabular}{|l|} 
double- \\
emulsion \\
(water-in- \\
oil-in-water) \\
solvent \\
evaporation
\end{tabular} & nanoparticl & $\begin{array}{l}\text { Chitosan, alginate, } \\
\text { mPEG-b-PLGA. }\end{array}$ & $\begin{array}{l}\text { in } \\
\text { vivo }\end{array}$ & $\begin{array}{l}\text { plasma } \\
\text { insulin level }\end{array}$ & $\begin{array}{l}\text { RQ1, } \\
\text { RQ2, } \\
\text { RQ3, RQ4 }\end{array}$ \\
\hline $\begin{array}{l}1 \\
9\end{array}$ & $\begin{array}{l}\text { Alsulay } \\
\text { s et al }\end{array}$ & $\begin{array}{l}20 \\
19\end{array}$ & \begin{tabular}{|l|} 
Dove \\
Medical \\
Press \\
Limited
\end{tabular} & $\begin{array}{l}\text { International } \\
\text { Journal of } \\
\text { Nanomedicin } \\
\text { e }(6,400)\end{array}$ & $\begin{array}{l}\text { double } \\
\text { emulsificatio } \\
n\end{array}$ & $\begin{array}{l}\text { Solid lipid } \\
\text { nanoparticl } \\
\text { e }\end{array}$ & $\begin{array}{l}\text { L-penetratin, D- } \\
\text { penetratin. }\end{array}$ & $\begin{array}{l}\text { in } \\
\text { vivo }\end{array}$ & $\begin{array}{l}\text { plasma } \\
\text { insulin level }\end{array}$ & $\begin{array}{l}\text { RQ1, } \\
\text { RQ2, } \\
\text { RQ3, RQ4 }\end{array}$ \\
\hline $\begin{array}{l}2 \\
0\end{array}$ & $\begin{array}{l}\text { Xie et } \\
\text { al }\end{array}$ & $\begin{array}{l}20 \\
18\end{array}$ & $\begin{array}{l}\text { Future } \\
\text { Medicine }\end{array}$ & $\begin{array}{l}\text { Nanomedicin } \\
\text { e (London) } \\
(4,727)\end{array}$ & $\mathrm{N} / \mathrm{A}$ & $\begin{array}{l}\text { polymerso } \\
\text { me }\end{array}$ & Pluronic p85, PLGA. & $\begin{array}{l}\text { in } \\
\text { vivo }\end{array}$ & $\begin{array}{l}\text { plasma } \\
\text { insulin level }\end{array}$ & RQ3, RQ4 \\
\hline $\begin{array}{l}2 \\
1\end{array}$ & $\begin{array}{l}\text { Sheng } \\
\text { et al }\end{array}$ & $\begin{array}{l}20 \\
15\end{array}$ & $\begin{array}{l}\text { ACS } \\
\text { Publicatio } \\
\text { ns }\end{array}$ & \begin{tabular}{|l} 
ACS Applied \\
Materials \& \\
Interfaces \\
$(8,758)$
\end{tabular} & \begin{tabular}{|l} 
double \\
emulsion \\
solvent \\
evaporation
\end{tabular} & $\begin{array}{l}\text { nanoparticl } \\
\text { e }\end{array}$ & $\begin{array}{l}\text { N-trymethyl chitosan, } \\
\text { PLGA. }\end{array}$ & $\begin{array}{l}\text { in } \\
\text { vivo }\end{array}$ & $\begin{array}{l}\text { glucose } \\
\text { insulin level }\end{array}$ & $\begin{array}{l}\text { RQ1, } \\
\text { RQ2, } \\
\text { RQ3, RQ4 }\end{array}$ \\
\hline $\begin{array}{l}2 \\
2\end{array}$ & $\begin{array}{l}\text { El- } \\
\text { Leithy } \\
\text { et al }\end{array}$ & $\begin{array}{l}20 \\
19\end{array}$ & Elsevier & \begin{tabular}{|l|} 
International \\
Journal of \\
Pharmaceutic \\
s $(5,875)$
\end{tabular} & $\begin{array}{l}\text { ionic } \\
\text { gelation }\end{array}$ & $\begin{array}{l}\text { nanoparticl } \\
\text { e }\end{array}$ & $\begin{array}{l}\text { chitosan, } \\
\text { tripolyphosphate } \\
\text { (TPP). }\end{array}$ & $\begin{array}{l}\text { in } \\
\text { vivo }\end{array}$ & $\begin{array}{l}\text { plasma } \\
\text { insulin level }\end{array}$ & $\begin{array}{l}\text { RQ1, } \\
\text { RQ2, } \\
\text { RQ3, RQ4 }\end{array}$ \\
\hline $\begin{array}{l}2 \\
3\end{array}$ & $\begin{array}{l}\text { Zeng et } \\
\text { al }\end{array}$ & $\begin{array}{l}20 \\
18\end{array}$ & $\begin{array}{l}\text { Wiley } \\
\text { Online } \\
\text { Library }\end{array}$ & $\begin{array}{l}\text { Advanced } \\
\text { Healthcare } \\
\text { Materials } \\
(7,367)\end{array}$ & $\mathrm{N} / \mathrm{A}$ & $\begin{array}{l}\text { nanoparticl } \\
\text { e }\end{array}$ & $\begin{array}{l}\text { DDAB } \\
\text { (dimethyldioctadecyla } \\
\text { mmonium bromide). }\end{array}$ & $\begin{array}{l}\text { in } \\
\text { vivo }\end{array}$ & $\begin{array}{l}\text { plasma } \\
\text { insulin level }\end{array}$ & RQ3, RQ4 \\
\hline $\begin{array}{l}2 \\
4\end{array}$ & $\begin{array}{l}\text { Verma } \\
\text { et al }\end{array}$ & $\begin{array}{l}20 \\
15\end{array}$ & Elsevier & \begin{tabular}{|l|} 
Acta \\
Biomaterialia \\
$(8,947)$
\end{tabular} & $\begin{array}{l}\text { microemulsi } \\
\text { on }\end{array}$ & $\begin{array}{l}\text { nanoparticl } \\
\text { e }\end{array}$ & vitamin B12, chitosan. & $\begin{array}{l}\text { in } \\
\text { vivo }\end{array}$ & $\begin{array}{l}\text { plasma } \\
\text { insulin level }\end{array}$ & \begin{tabular}{|l|} 
RQ1, \\
RQ2, \\
RQ3, RQ4
\end{tabular} \\
\hline $\begin{array}{l}2 \\
5\end{array}$ & $\begin{array}{l}\text { Tian et } \\
\text { al }\end{array}$ & $\begin{array}{l}20 \\
18\end{array}$ & $\begin{array}{l}\text { Wiley } \\
\text { Online } \\
\text { Library }\end{array}$ & $\begin{array}{l}\text { Advanced } \\
\text { Healthcare } \\
\text { Materials }\end{array}$ & $\begin{array}{l}\text { two-step } \\
\text { flash } \\
\text { nanocomple }\end{array}$ & $\begin{array}{l}\text { nanoparticl } \\
\text { e }\end{array}$ & $\begin{array}{l}\text { Hyaluronic acid, } \\
\text { thiolated hyaluronic } \\
\text { acid. }\end{array}$ & $\begin{array}{l}\text { in } \\
\text { vivo }\end{array}$ & $\begin{array}{l}\text { plasma } \\
\text { insulin level }\end{array}$ & $\begin{array}{l}\text { RQ1, } \\
\text { RQ2, } \\
\text { RQ3, RQ4 }\end{array}$ \\
\hline
\end{tabular}




\begin{tabular}{|c|c|c|c|c|c|c|c|c|c|c|}
\hline $\begin{array}{l}\mathrm{N} \\
\mathrm{o}\end{array}$ & Author & $\begin{array}{l}\text { Yea } \\
\mathrm{r}\end{array}$ & Publisher & $\begin{array}{l}\text { Journal and } \\
\text { Impact Factor }\end{array}$ & Method & $\begin{array}{l}\text { Delivery } \\
\text { System }\end{array}$ & Formulation & $\begin{array}{l}\text { Test } \\
\text { Meth } \\
\text { od }\end{array}$ & \begin{tabular}{|l} 
BA \\
Parameters
\end{tabular} & $\begin{array}{l}\text { Theme } \\
\text { Classifica } \\
\text { tion }\end{array}$ \\
\hline & & & & $(7,367)$ & xation & & & & & \\
\hline $\begin{array}{l}2 \\
6\end{array}$ & $\begin{array}{l}\text { Malath } \\
\text { i et al }\end{array}$ & $\begin{array}{l}20 \\
15\end{array}$ & \begin{tabular}{|l} 
Dove \\
Medical \\
Press \\
Limited
\end{tabular} & $\begin{array}{l}\text { International } \\
\text { Journal of } \\
\text { Nanomedicin } \\
\text { e }(6,400)\end{array}$ & $\begin{array}{l}\text { emulsion- } \\
\text { solvent } \\
\text { evaporation }\end{array}$ & nanoparticl & $\begin{array}{l}\text { d- } \alpha \text {-tocopherol } \\
\text { poly(ethylene glycol) } \\
1000 \text { succinate (TPGS), } \\
\text { PLGA, PEG. }\end{array}$ & in & $\begin{array}{l}\text { plasma } \\
\text { insulin level }\end{array}$ & $\begin{array}{l}\text { RQ1, } \\
\text { RQ2, } \\
\text { RQ3, RQ4 }\end{array}$ \\
\hline $\begin{array}{l}2 \\
7\end{array}$ & $\begin{array}{l}\text { Wang } \\
\text { et al }\end{array}$ & $\begin{array}{l}20 \\
17\end{array}$ & Elsevier & \begin{tabular}{l|} 
Carbohydrate \\
Polymers \\
$(9.381)$
\end{tabular} & $\mathrm{N} / \mathrm{A}$ & $\begin{array}{l}\text { nanoparticl } \\
\text { e }\end{array}$ & $\begin{array}{l}\text { Deacetylated chitosan, } \\
\text { Carboxymethyl } \\
\text { chitosan }\end{array}$ & $\begin{array}{l}\text { in } \\
\text { vivo }\end{array}$ & $\begin{array}{l}\text { plasma } \\
\text { insulin level }\end{array}$ & RQ3, RQ4 \\
\hline $\begin{array}{l}2 \\
8\end{array}$ & $\begin{array}{l}\text { Sun et } \\
\text { al }\end{array}$ & $\begin{array}{l}20 \\
16\end{array}$ & MDPI & $\begin{array}{l}\text { International } \\
\text { Journal of } \\
\text { Molecular } \\
\text { Sciences } \\
(5.923)\end{array}$ & $\begin{array}{l}\text { emulsion } \\
\text { solvent } \\
\text { diffusion }\end{array}$ & nanoparticl & $\begin{array}{l}\text { Sodium deoxycholate, } \\
\text { PLGA, Hydroxypropyl } \\
\text { methyl cellulose } \\
\text { phthalate (HP55). }\end{array}$ & $\begin{array}{l}\text { in } \\
\text { vivo }\end{array}$ & $\begin{array}{l}\text { glucose } \\
\text { insulin level }\end{array}$ & $\begin{array}{l}\text { RQ1, } \\
\text { RQ2, } \\
\text { RQ3, RQ4 }\end{array}$ \\
\hline $\begin{array}{l}2 \\
9\end{array}$ & $\begin{array}{l}\text { Liu et } \\
\text { al }\end{array}$ & $\begin{array}{l}20 \\
16\end{array}$ & Elsevier & $\begin{array}{l}\text { Journal of } \\
\text { Controlled } \\
\text { Release } \\
(9,776)\end{array}$ & \begin{tabular}{|l|} 
Ionotropic \\
gelation and \\
polyelectroly \\
te complex
\end{tabular} & $\begin{array}{l}\text { nanoparticl } \\
\text { e }\end{array}$ & $\begin{array}{l}\text { TMC, sodium } \\
\text { tripolyphosphate } \\
\text { (TPP), Chitosan. }\end{array}$ & $\begin{array}{l}\text { in } \\
\text { vivo }\end{array}$ & $\begin{array}{l}\text { plasma } \\
\text { insulin level }\end{array}$ & $\begin{array}{l}\text { RQ1, } \\
\text { RQ2, } \\
\text { RQ3, RQ4 }\end{array}$ \\
\hline $\begin{array}{l}3 \\
0\end{array}$ & $\begin{array}{l}\text { Fang et } \\
\text { al }\end{array}$ & $\begin{array}{l}20 \\
18\end{array}$ & Elsevier & \begin{tabular}{|l|} 
Journal of \\
Pharmaceutic \\
al Sciences \\
$(3.534)$
\end{tabular} & \begin{tabular}{|l|} 
spontaneous \\
emulsion \\
solvent \\
diffusion
\end{tabular} & nanoparticl & $\begin{array}{l}\text { hidroksipropil } \\
\text { metilselulosa ftalat } \\
\text { (HPMCP), PVA. }\end{array}$ & $\begin{array}{l}\text { in } \\
\text { vivo }\end{array}$ & $\begin{array}{l}\text { glucose } \\
\text { insulin level }\end{array}$ & $\begin{array}{l}\text { RQ1, } \\
\text { RQ2, } \\
\text { RQ3, RQ4 }\end{array}$ \\
\hline $\begin{array}{l}3 \\
1\end{array}$ & $\begin{array}{l}\text { Chen } \\
\text { et al }\end{array}$ & $\begin{array}{l}20 \\
19\end{array}$ & Elsevier & \begin{tabular}{|l|} 
International \\
Journal of \\
Pharmaceutic \\
s $(5,875)$
\end{tabular} & $\mathrm{N} / \mathrm{A}$ & $\begin{array}{l}\text { nanoparticl } \\
\text { e }\end{array}$ & $\begin{array}{l}\text { Chitosan /alginate, } \\
\text { Cp1-11 }\end{array}$ & $\begin{array}{l}\text { in } \\
\text { vivo }\end{array}$ & $\begin{array}{l}\text { plasma } \\
\text { insulin level }\end{array}$ & RQ3, RQ4 \\
\hline $\begin{array}{l}3 \\
2\end{array}$ & $\begin{array}{l}\text { Zheng } \\
\text { et al }\end{array}$ & $\begin{array}{l}20 \\
18\end{array}$ & $\begin{array}{l}\text { ACS } \\
\text { Publicatio } \\
\text { ns }\end{array}$ & $\begin{array}{l}\text { ACS Applied } \\
\text { Materials \& } \\
\text { Interfaces } \\
(8,758)\end{array}$ & $\begin{array}{l}\text { One step } \\
\text { nanoprecipit } \\
\text { ation }\end{array}$ & nanoparticl & EGP peptide, PLGA & $\begin{array}{l}\text { in } \\
\text { vivo }\end{array}$ & $\begin{array}{l}\text { plasma } \\
\text { insulin level }\end{array}$ & $\begin{array}{l}\text { RQ1, } \\
\text { RQ2, } \\
\text { RQ3, RQ4 }\end{array}$ \\
\hline $\begin{array}{l}3 \\
3\end{array}$ & $\begin{array}{l}\text { He et } \\
\text { al }\end{array}$ & $\begin{array}{l}20 \\
17\end{array}$ & Elsevier & $\begin{array}{l}\text { Biomaterials } \\
(12.479)\end{array}$ & $\begin{array}{l}\text { Flash } \\
\text { nanocomple } \\
\text { xation }\end{array}$ & $\begin{array}{l}\text { nanoparticl } \\
\text { e }\end{array}$ & $\begin{array}{l}\text { Chitosan, tripolifosfat } \\
\text { (TPP) }\end{array}$ & $\begin{array}{l}\text { in } \\
\text { vivo }\end{array}$ & $\begin{array}{l}\text { plasma } \\
\text { insulin level }\end{array}$ & $\begin{array}{l}\text { RQ1, } \\
\text { RQ2, } \\
\text { RQ3, RQ4 }\end{array}$ \\
\hline $\begin{array}{l}3 \\
4\end{array}$ & Li et al & $\begin{array}{l}20 \\
17\end{array}$ & Elsevier & $\begin{array}{l}\text { Materials } \\
\text { Science and } \\
\text { Engineering: } \\
\text { C (7.328) }\end{array}$ & $\mathrm{N} / \mathrm{A}$ & nanoparticl & $\begin{array}{l}\text { Chitosan modified by } \\
\text { L-valin }\end{array}$ & $\begin{array}{l}\text { in } \\
\text { vivo }\end{array}$ & $\begin{array}{l}\text { plasma } \\
\text { insulin level }\end{array}$ & RQ3. RQ4 \\
\hline $\begin{array}{l}3 \\
5\end{array}$ & \begin{tabular}{|l|} 
Bahma \\
n et al
\end{tabular} & $\begin{array}{l}20 \\
20\end{array}$ & MDPI & $\begin{array}{l}\text { Pharmaceutic } \\
\text { s (6.321) }\end{array}$ & $\mathrm{N} / \mathrm{A}$ & micelles & $\begin{array}{l}\text { Poly(styrene-co-maleic } \\
\text { acid) }\end{array}$ & $\begin{array}{l}\text { in } \\
\text { vivo }\end{array}$ & \begin{tabular}{|l|} 
glucose \\
insulin level
\end{tabular} & RQ3, RQ4 \\
\hline $\begin{array}{l}3 \\
6\end{array}$ & $\begin{array}{l}\text { Hu et } \\
\text { al }\end{array}$ & $\begin{array}{l}20 \\
19\end{array}$ & NCBI & \begin{tabular}{|l|} 
International \\
Journal of \\
Nanomedicin \\
e $(6,400)$
\end{tabular} & $\begin{array}{l}\text { anhydrous } \\
\text { co-solvent } \\
\text { lyophilizatio } \\
\mathrm{n}\end{array}$ & nanoemulsi & $\begin{array}{l}\text { Phospolipid (Oleic Acid } \\
\text { (OA), Ethyl Oleate (EO), } \\
\text { Isopropyl Myristate } \\
\text { (IPM)). }\end{array}$ & in & $\begin{array}{l}\text { plasma } \\
\text { insulin level }\end{array}$ & $\begin{array}{l}\text { RQ1, } \\
\text { RQ2, } \\
\text { RQ3, RQ4 }\end{array}$ \\
\hline $\begin{array}{l}3 \\
7\end{array}$ & $\begin{array}{l}\text { Fan et } \\
\text { al }\end{array}$ & $\begin{array}{l}20 \\
18\end{array}$ & Elsevier & \begin{tabular}{|l} 
Biomaterials \\
$(12.479)$
\end{tabular} & $\mathrm{N} / \mathrm{A}$ & \begin{tabular}{|l|} 
nanoparticl \\
$\mathrm{e}$
\end{tabular} & $\begin{array}{l}\text { Deoxycholic acid, } \\
\text { chloroquine (CQ), PGA. }\end{array}$ & $\begin{array}{l}\text { in } \\
\text { vivo }\end{array}$ & \begin{tabular}{|l|} 
plasma \\
insulin level
\end{tabular} & RQ3, RQ4 \\
\hline $\begin{array}{l}3 \\
8\end{array}$ & $\begin{array}{l}\text { Sun et } \\
\text { al }\end{array}$ & $\begin{array}{l}20 \\
18\end{array}$ & $\begin{array}{l}\text { ACS } \\
\text { Publicatio } \\
\text { ns }\end{array}$ & $\begin{array}{l}\text { Biomacromol } \\
\text { ecules (6.988) }\end{array}$ & $\mathrm{N} / \mathrm{A}$ & $\begin{array}{l}\text { nanoparticl } \\
\text { e }\end{array}$ & $\begin{array}{l}\text { Chitosan, eudragit } \\
\text { S100, transcriptional } \\
\text { peptide (tat) }\end{array}$ & $\begin{array}{l}\text { in } \\
\text { vivo }\end{array}$ & \begin{tabular}{|l|} 
plasma \\
insulin level, \\
glucose \\
insulin level
\end{tabular} & RQ3, RQ4 \\
\hline $\begin{array}{l}3 \\
9\end{array}$ & $\begin{array}{l}\text { Shrest } \\
\text { ha et al }\end{array}$ & $\begin{array}{l}20 \\
16\end{array}$ & $\begin{array}{l}\text { Wiley } \\
\text { Online } \\
\text { Library }\end{array}$ & $\begin{array}{l}\text { Advanced } \\
\text { Functional } \\
\text { Materials } \\
(18.808)\end{array}$ & $\mathrm{N} / \mathrm{A}$ & $\begin{array}{l}\text { nanoparticl } \\
\text { e }\end{array}$ & Chitosan & $\begin{array}{l}\text { in } \\
\text { vivo }\end{array}$ & $\begin{array}{l}\text { plasma } \\
\text { insulin level }\end{array}$ & RQ3, RQ4 \\
\hline $\begin{array}{l}4 \\
0\end{array}$ & $\begin{array}{l}\text { Fukuo } \\
\text { ka et al }\end{array}$ & $\begin{array}{l}20 \\
18\end{array}$ & $\begin{array}{l}\text { The } \\
\text { Pharmace } \\
\text { utical } \\
\text { Society of }\end{array}$ & $\begin{array}{l}\text { Biological and } \\
\text { Pharmaceutic } \\
\text { al Bulletin } \\
(2.233)\end{array}$ & $\mathrm{N} / \mathrm{A}$ & $\begin{array}{l}\text { nanoparticl } \\
\mathrm{e}\end{array}$ & Oligoarginin, CPP R6 & $\begin{array}{l}\text { in } \\
\text { vivo }\end{array}$ & \begin{tabular}{|l|} 
plasma \\
insulin level, \\
glucose \\
insulin level
\end{tabular} & RQ3, RQ4 \\
\hline
\end{tabular}




\begin{tabular}{|c|c|c|c|c|c|c|c|c|c|c|}
\hline $\begin{array}{l}\mathrm{N} \\
\mathrm{o}\end{array}$ & Author & $\begin{array}{l}\text { Yea } \\
\mathrm{r}\end{array}$ & Publisher & $\begin{array}{l}\text { Journal and } \\
\text { Impact Factor }\end{array}$ & Method & $\begin{array}{l}\text { Delivery } \\
\text { System }\end{array}$ & Formulation & $\begin{array}{l}\text { Test } \\
\text { Meth } \\
\text { od }\end{array}$ & $\begin{array}{l}\text { BA } \\
\text { Parameters }\end{array}$ & $\begin{array}{l}\text { Theme } \\
\text { Classifica } \\
\text { tion }\end{array}$ \\
\hline & & & Japan & & & & & & & \\
\hline $\begin{array}{l}4 \\
1\end{array}$ & $\begin{array}{l}\text { Zhang } \\
\text { et al }\end{array}$ & $\begin{array}{l}20 \\
17\end{array}$ & $\begin{array}{l}\text { ACS } \\
\text { Publicatio } \\
\text { ns }\end{array}$ & $\begin{array}{l}\text { Biomacromol } \\
\text { ecules (6.988) }\end{array}$ & $\begin{array}{l}\mathrm{W} / \mathrm{O} / \mathrm{W} \\
\text { solvent } \\
\text { evaporation }\end{array}$ & $\begin{array}{l}\text { nanoparticl } \\
\text { e }\end{array}$ & PLGA, Chitosan. & $\begin{array}{l}\text { in } \\
\text { vivo }\end{array}$ & $\begin{array}{l}\text { glucose } \\
\text { insulin level }\end{array}$ & RQ3, RQ4 \\
\hline $\begin{array}{l}4 \\
2\end{array}$ & $\begin{array}{l}\text { Wu et } \\
\text { al }\end{array}$ & $\begin{array}{l}20 \\
18\end{array}$ & $\begin{array}{l}\text { ACS } \\
\text { Publicatio } \\
\text { ns }\end{array}$ & $\begin{array}{l}\text { ACS Applied } \\
\text { Materials \& } \\
\text { Interfaces } \\
(8,758)\end{array}$ & \begin{tabular}{|l} 
self- \\
assembly \\
nanoprecipit \\
ation
\end{tabular} & 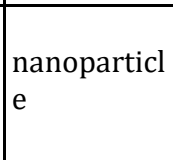 & PLGA, oktaarginin. & $\begin{array}{l}\text { in } \\
\text { vivo }\end{array}$ & $\begin{array}{l}\text { plasma } \\
\text { insulin level }\end{array}$ & RQ3, RQ4 \\
\hline $\begin{array}{l}4 \\
3\end{array}$ & $\begin{array}{l}\text { Wu et } \\
\text { al }\end{array}$ & $\begin{array}{l}20 \\
17\end{array}$ & Elsevier & $\begin{array}{l}\text { Journal of } \\
\text { Controlled } \\
\text { Release } \\
(9,776)\end{array}$ & $\begin{array}{l}\text { self- } \\
\text { assembly } \\
\text { nanoprecipit } \\
\text { ation }\end{array}$ & nanoparticl & Butyrate & $\begin{array}{l}\text { in } \\
\text { vivo }\end{array}$ & $\begin{array}{l}\text { plasma } \\
\text { insulin level }\end{array}$ & RQ3, RQ4 \\
\hline $\begin{array}{l}4 \\
4\end{array}$ & $\begin{array}{l}\text { He et } \\
\text { al }\end{array}$ & $\begin{array}{l}20 \\
15\end{array}$ & Elsevier & $\begin{array}{l}\text { International } \\
\text { Journal of } \\
\text { Pharmaceutic } \\
\text { s }(5,875)\end{array}$ & $\begin{array}{l}\text { combination } \\
\text { of double } \\
\text { emulsion } \\
\text { and solvent- } \\
\text { evaporation } \\
\text { and a } \\
\text { thermal- } \\
\text { sensitive } \\
\text { hydrogel }\end{array}$ & $\begin{array}{l}\text { solid lipid } \\
\text { nanoparticl } \\
\text { e }\end{array}$ & Vitamin B12 & $\begin{array}{l}\text { in } \\
\text { vivo }\end{array}$ & $\begin{array}{l}\text { glucose } \\
\text { insulin level }\end{array}$ & $\begin{array}{l}\text { RQ1, } \\
\text { RQ2, } \\
\text { RQ3, RQ4 }\end{array}$ \\
\hline $\begin{array}{l}4 \\
5\end{array}$ & $\begin{array}{l}\text { Sahoo } \\
\text { et al }\end{array}$ & $\begin{array}{l}20 \\
19\end{array}$ & Elsevier & \begin{tabular}{|l} 
Materials \\
Today \\
Communicati \\
ons (3.383)
\end{tabular} & $\mathrm{N} / \mathrm{A}$ & particle & Chitosan & $\begin{array}{l}\text { in } \\
\text { vivo }\end{array}$ & $\begin{array}{l}\text { plasma } \\
\text { insulin level }\end{array}$ & RQ3, RQ4 \\
\hline $\begin{array}{l}4 \\
6\end{array}$ & $\begin{array}{l}\text { Shan et } \\
\text { al }\end{array}$ & $\begin{array}{l}20 \\
16\end{array}$ & $\begin{array}{l}\text { ACS } \\
\text { Publicatio } \\
\text { ns }\end{array}$ & \begin{tabular}{|l} 
ACS Applied \\
Materials \& \\
Interfaces \\
$(8,758)$
\end{tabular} & $\begin{array}{l}\text { self- } \\
\text { assembly } \\
\text { nanoprecipit } \\
\text { ation }\end{array}$ & nanoparticl & PVA & $\begin{array}{l}\text { in } \\
\text { vivo }\end{array}$ & $\begin{array}{l}\text { plasma } \\
\text { insulin level }\end{array}$ & RQ3, RQ4 \\
\hline $\begin{array}{l}4 \\
7\end{array}$ & $\begin{array}{l}\text { Sheng } \\
\text { et al }\end{array}$ & $\begin{array}{l}20 \\
16\end{array}$ & Elsevier & $\begin{array}{l}\text { Journal of } \\
\text { Controlled } \\
\text { Release } \\
(9,776)\end{array}$ & $\mathrm{N} / \mathrm{A}$ & $\begin{array}{l}\text { nanoparticl } \\
\text { e }\end{array}$ & PLGA, Chitosan. & $\begin{array}{l}\text { in } \\
\text { vivo }\end{array}$ & $\begin{array}{l}\text { plasma } \\
\text { insulin level }\end{array}$ & RQ3, RQ4 \\
\hline $\begin{array}{l}4 \\
8\end{array}$ & $\begin{array}{l}\text { Elsaye } \\
\text { d et al }\end{array}$ & $\begin{array}{l}20 \\
18\end{array}$ & MDPI & $\begin{array}{l}\text { Marine Drugs } \\
(5.118)\end{array}$ & N/A & $\begin{array}{l}\text { nanoparticl } \\
\text { e }\end{array}$ & Chitosan, oleic acid. & $\begin{array}{l}\text { in } \\
\text { vivo }\end{array}$ & $\begin{array}{l}\text { plasma } \\
\text { insulin level }\end{array}$ & RQ3, RQ4 \\
\hline $\begin{array}{l}4 \\
9\end{array}$ & $\begin{array}{l}\text { Mumu } \\
\text { ni et al }\end{array}$ & $\begin{array}{l}20 \\
20\end{array}$ & Elsevier & $\begin{array}{l}\text { Carbohydrate } \\
\text { Polymers } \\
(9.381)\end{array}$ & Self-gelation & $\begin{array}{l}\text { nanoparticl } \\
\text { e }\end{array}$ & $\begin{array}{l}\text { Chitosan, } \\
\text { aqueous soluble snail } \\
\text { mucin }\end{array}$ & $\begin{array}{l}\text { in } \\
\text { vivo }\end{array}$ & $\begin{array}{l}\text { plasma } \\
\text { insulin level }\end{array}$ & $\begin{array}{l}\text { RQ1, } \\
\text { RQ2, } \\
\text { RQ3, RQ4 }\end{array}$ \\
\hline $\begin{array}{l}5 \\
0\end{array}$ & $\begin{array}{l}\text { Yan et } \\
\text { al }\end{array}$ & $\begin{array}{l}20 \\
19\end{array}$ & Springer & $\begin{array}{l}\text { AAPS } \\
\text { PharmSciTec } \\
\text { h }(3,246)\end{array}$ & $\mathrm{N} / \mathrm{A}$ & $\begin{array}{l}\text { nanoparticl } \\
\text { e }\end{array}$ & Chitosan & in & $\begin{array}{l}\text { plasma } \\
\text { insulin level }\end{array}$ & $\begin{array}{l}\text { RQ1, } \\
\text { RQ2, } \\
\text { RQ3, RQ4 }\end{array}$ \\
\hline $\begin{array}{l}5 \\
1\end{array}$ & $\begin{array}{l}\text { Boushr } \\
\text { a et al }\end{array}$ & $\begin{array}{l}20 \\
16\end{array}$ & Elsevier & $\begin{array}{l}\text { International } \\
\text { Journal of } \\
\text { Pharmaceutic } \\
\text { s }(5,875)\end{array}$ & $\begin{array}{l}\text { emulsificatio } \\
\text { n solvent- } \\
\text { evaporation } \\
\text { technique to } \\
\text { form double } \\
\text { emulsion } \\
(w / o / w)\end{array}$ & $\begin{array}{l}\text { solid lipid } \\
\text { nanoparticl } \\
\text { e }\end{array}$ & $\begin{array}{l}\text { propilen glikol (PG), } \\
\text { polietilen glikol (PEG) } \\
\text { 400, PEG } 600 .\end{array}$ & in & $\begin{array}{l}\text { glucose } \\
\text { insulin level }\end{array}$ & $\begin{array}{l}\text { RQ1, } \\
\text { RQ2, } \\
\text { RQ3, RQ4 }\end{array}$ \\
\hline $\begin{array}{l}5 \\
2\end{array}$ & $\begin{array}{l}\text { Niu et } \\
\text { al }\end{array}$ & $\begin{array}{l}20 \\
17\end{array}$ & Elsevier & $\begin{array}{l}\text { Journal of } \\
\text { Controlled } \\
\text { Release } \\
(9,776)\end{array}$ & $\begin{array}{l}\text { modified } \\
\text { solvent } \\
\text { displacemen } \\
\mathrm{t}\end{array}$ & $\begin{array}{l}\text { nanocapsul } \\
\text { e }\end{array}$ & Polyarginine & $\begin{array}{l}\text { in } \\
\text { vivo }\end{array}$ & $\begin{array}{l}\text { glucose } \\
\text { insulin level }\end{array}$ & $\begin{array}{l}\text { RQ1, } \\
\text { RQ2, } \\
\text { RQ3, RQ4 }\end{array}$ \\
\hline $\begin{array}{l}5 \\
3\end{array}$ & $\begin{array}{l}\text { Omid } \\
\text { et al }\end{array}$ & $\begin{array}{l}20 \\
17\end{array}$ & Elsevier & $\begin{array}{l}\text { International } \\
\text { Journal of } \\
\text { Pharmaceutic } \\
\text { s }(5,875)\end{array}$ & $\mathrm{N} / \mathrm{A}$ & $\begin{array}{l}\text { nanoparticl } \\
\text { e }\end{array}$ & $\begin{array}{l}\text { Chitosan, 1-ethyl-3-(3- } \\
\text { dimethylaminopropyl) } \\
\text { carbodiimide (EDC). }\end{array}$ & $\begin{array}{l}\text { in } \\
\text { vivo }\end{array}$ & $\begin{array}{l}\text { plasma } \\
\text { insulin level }\end{array}$ & RQ3, RQ4 \\
\hline
\end{tabular}




\begin{tabular}{|c|c|c|c|c|c|c|c|c|c|c|}
\hline $\begin{array}{l}\mathrm{N} \\
\mathrm{o}\end{array}$ & Author & $\begin{array}{l}\text { Yea } \\
\text { r }\end{array}$ & Publisher & $\begin{array}{l}\text { Journal and } \\
\text { Impact Factor }\end{array}$ & Method & $\begin{array}{l}\text { Delivery } \\
\text { System }\end{array}$ & Formulation & $\begin{array}{l}\text { Test } \\
\text { Meth } \\
\text { od }\end{array}$ & $\begin{array}{l}\text { BA } \\
\text { Parameters }\end{array}$ & $\begin{array}{l}\text { Theme } \\
\text { Classifica } \\
\text { tion }\end{array}$ \\
\hline $\begin{array}{l}5 \\
4\end{array}$ & $\begin{array}{l}\text { Zhang } \\
\text { et al }\end{array}$ & $\begin{array}{l}20 \\
21\end{array}$ & $\begin{array}{l}\text { ACS } \\
\text { Publicatio } \\
\text { ns }\end{array}$ & $\begin{array}{l}\text { ACS Applied } \\
\text { Materials \& } \\
\text { Interfaces } \\
(8,758)\end{array}$ & $\mathrm{N} / \mathrm{A}$ & $\begin{array}{l}\text { silica } \\
\text { nanoparticl } \\
\text { e }\end{array}$ & $\begin{array}{l}\text { Cetyltrimethylammoni } \\
\text { um bromide, } \\
\text { Tetraethyl silicate, } \\
1,3,5- \\
\text { trimethylbenzene, } \\
\text { Nhydroxysuccinimide. }\end{array}$ & $\begin{array}{l}\text { in } \\
\text { vivo }\end{array}$ & $\begin{array}{l}\text { glucose } \\
\text { insulin level }\end{array}$ & RQ3, RQ4 \\
\hline $\begin{array}{l}5 \\
5\end{array}$ & $\begin{array}{l}\text { Zhou } \\
\text { et al }\end{array}$ & $\begin{array}{l}20 \\
20\end{array}$ & Springer & $\begin{array}{l}\text { Journal of } \\
\text { Nanobiotechn } \\
\text { ology } \\
(10.435)\end{array}$ & $\mathrm{N} / \mathrm{A}$ & $\begin{array}{l}\text { nanoparticl } \\
\text { e }\end{array}$ & Sodium alginate (ALG) & $\begin{array}{l}\text { in } \\
\text { vivo }\end{array}$ & $\begin{array}{l}\text { glucose } \\
\text { insulin level }\end{array}$ & RQ3, RQ4 \\
\hline $\begin{array}{l}5 \\
6\end{array}$ & $\begin{array}{l}\text { Winart } \\
\text { i et al }\end{array}$ & $\begin{array}{l}20 \\
18\end{array}$ & \begin{tabular}{|l|} 
Indonesian \\
Journal of \\
Pharmacy
\end{tabular} & $\begin{array}{l}\text { Indonesian } \\
\text { Journal of } \\
\text { Pharmacy } \\
(0,56)\end{array}$ & $\mathrm{N} / \mathrm{A}$ & \begin{tabular}{|l} 
self- \\
nanoemulsi \\
fying drug \\
delivery \\
system \\
(SNEDDS)
\end{tabular} & $\begin{array}{l}\text { miglyol 812N, tween } \\
\text { 80, propylene glycol. }\end{array}$ & $\begin{array}{l}\text { in } \\
\text { vivo }\end{array}$ & $\begin{array}{l}\text { plasma } \\
\text { insulin level }\end{array}$ & RQ3, RQ4 \\
\hline $\begin{array}{l}5 \\
7\end{array}$ & $\begin{array}{l}\text { Liu et } \\
\text { al }\end{array}$ & $\begin{array}{l}20 \\
19\end{array}$ & Elsevier & $\begin{array}{l}\text { International } \\
\text { Journal of } \\
\text { Pharmaceutic } \\
\text { s }(5,875)\end{array}$ & $\mathrm{N} / \mathrm{A}$ & $\begin{array}{l}\text { nanocompl } \\
\text { ex }\end{array}$ & Chitosan & $\begin{array}{l}\text { in } \\
\text { vivo }\end{array}$ & $\begin{array}{l}\text { glucose } \\
\text { insulin level }\end{array}$ & $\begin{array}{l}\text { RQ1, } \\
\text { RQ2, } \\
\text { RQ3, RQ4 }\end{array}$ \\
\hline $\begin{array}{l}5 \\
8\end{array}$ & $\begin{array}{l}\text { Zhang } \\
\text { et al }\end{array}$ & $\begin{array}{l}20 \\
18\end{array}$ & $\begin{array}{l}\text { Royal } \\
\text { Society of } \\
\text { Chemistry }\end{array}$ & \begin{tabular}{|l} 
Journal of \\
Materials \\
Chemistry B \\
$(6.331)$
\end{tabular} & N/A & $\begin{array}{l}\text { nanoparticl } \\
\text { e }\end{array}$ & $\begin{array}{l}\text { PGLA, alginate, } \\
\text { chitosan. }\end{array}$ & $\begin{array}{l}\text { in } \\
\text { vivo }\end{array}$ & $\begin{array}{l}\text { glucose } \\
\text { insulin level }\end{array}$ & RQ3, RQ4 \\
\hline $\begin{array}{l}5 \\
9\end{array}$ & $\begin{array}{l}\text { Guo et } \\
\text { al }\end{array}$ & $\begin{array}{l}20 \\
16\end{array}$ & $\begin{array}{l}\text { Taylor \& } \\
\text { Francis }\end{array}$ & $\begin{array}{l}\text { Drug Delivery } \\
(3.095)\end{array}$ & $\begin{array}{l}\text { emulsion- } \\
\text { solvent } \\
\text { evaporation }\end{array}$ & $\begin{array}{l}\text { nanoparticl } \\
\text { e }\end{array}$ & $\begin{array}{l}\text { PLGA; Stearyl-Tat (Ste- } \\
\text { Tat); N,N,N-trimethyl- } \\
\text { N-dodecyl chitosan. }\end{array}$ & $\begin{array}{l}\text { in } \\
\text { vivo }\end{array}$ & $\begin{array}{l}\text { plasma } \\
\text { insulin level }\end{array}$ & $\begin{array}{l}\text { RQ1, } \\
\text { RQ2, } \\
\text { RQ3, RQ4 }\end{array}$ \\
\hline $\begin{array}{l}6 \\
0\end{array}$ & $\begin{array}{l}\text { Liu et } \\
\text { al }\end{array}$ & $\begin{array}{l}20 \\
19\end{array}$ & Elsevier & $\begin{array}{l}\text { International } \\
\text { Journal of } \\
\text { Pharmaceutic } \\
\text { s }(5,875)\end{array}$ & $\mathrm{N} / \mathrm{A}$ & \begin{tabular}{|l|} 
self- \\
emulsifying \\
drug \\
delivery \\
systems \\
(SEDDSs)
\end{tabular} & $\begin{array}{l}\text { Monoacyl } \\
\text { phosphatidylcholine } \\
\text { (MAPC), Labrasol } \\
\text { (LAB). }\end{array}$ & $\begin{array}{l}\text { in } \\
\text { vivo }\end{array}$ & $\begin{array}{l}\text { glucose } \\
\text { insulin level }\end{array}$ & $\begin{array}{l}\text { RQ1, } \\
\text { RQ2, } \\
\text { RQ3, RQ4 }\end{array}$ \\
\hline $\begin{array}{l}6 \\
1\end{array}$ & $\begin{array}{l}\text { Ansari } \\
\text { et al }\end{array}$ & $\begin{array}{l}20 \\
16\end{array}$ & $\begin{array}{l}\text { Taylor \& } \\
\text { Francis }\end{array}$ & $\begin{array}{l}\text { Drug Delivery } \\
(3.095)\end{array}$ & \begin{tabular}{|l|} 
double \\
emulsion \\
solvent \\
evaporation \\
(w/o/w) \\
technique
\end{tabular} & $\begin{array}{l}\text { solid lipid } \\
\text { nanoparticl } \\
\text { es }\end{array}$ & $\begin{array}{l}\text { Dynasan 14, Soya } \\
\text { lecithin, polyvinyl } \\
\text { alcohol (PVA), PLGA, } \\
\text { eudragit }\end{array}$ & $\begin{array}{l}\text { in } \\
\text { vivo }\end{array}$ & $\begin{array}{l}\text { plasma } \\
\text { insulin level }\end{array}$ & RQ3, RQ4 \\
\hline $\begin{array}{l}6 \\
2\end{array}$ & $\begin{array}{l}\text { Sun et } \\
\text { al }\end{array}$ & $\begin{array}{l}20 \\
15\end{array}$ & $\begin{array}{l}\text { Dove } \\
\text { Medical } \\
\text { Press } \\
\text { Limited }\end{array}$ & $\begin{array}{l}\text { International } \\
\text { Journal of } \\
\text { Nanomedicin } \\
\text { e }(6,400)\end{array}$ & $\begin{array}{l}\text { emulsion } \\
\text { solvent } \\
\text { diffusion }\end{array}$ & $\begin{array}{l}\text { nanoparticl } \\
\text { e }\end{array}$ & $\begin{array}{l}\text { Poly- vinyl alcohol, } \\
\text { PLGA, Eudragit } \AA \text { FS } \\
\text { 30D }\end{array}$ & $\begin{array}{l}\text { in } \\
\text { vivo }\end{array}$ & $\begin{array}{l}\text { plasma } \\
\text { insulin level }\end{array}$ & RQ3, RQ4 \\
\hline $\begin{array}{l}6 \\
3\end{array}$ & $\begin{array}{l}\text { Alfaro } \\
\text { et al }\end{array}$ & $\begin{array}{l}20 \\
20\end{array}$ & Elsevier & $\begin{array}{l}\text { Journal of } \\
\text { Drug Delivery } \\
\text { Science and } \\
\text { Technology } \\
(3,981)\end{array}$ & $\mathrm{N} / \mathrm{A}$ & $\begin{array}{l}\text { self- } \\
\text { nanoemulsi } \\
\text { fying }\end{array}$ & $\begin{array}{l}\text { Lauroglycol FCC, } \\
\text { surfactant (Cremophor } \\
\text { EL) and co-surfactant } \\
\text { (Labrafil M1944CS) }\end{array}$ & $\begin{array}{l}\text { in } \\
\text { vivo }\end{array}$ & $\begin{array}{l}\text { plasma } \\
\text { insulin level }\end{array}$ & $\begin{array}{l}\text { RQ1, } \\
\text { RQ2, } \\
\text { RQ3, RQ4 }\end{array}$ \\
\hline $\begin{array}{l}6 \\
4\end{array}$ & $\begin{array}{l}\text { Koland } \\
\text { et al }\end{array}$ & $\begin{array}{l}20 \\
21\end{array}$ & IJPER & $\begin{array}{l}\text { Indian } \\
\text { Journal of } \\
\text { Pharmaceutic } \\
\text { al Education } \\
\text { and Research } \\
(0.425)\end{array}$ & $\begin{array}{l}\text { modified } \\
\text { solvent } \\
\text { emulsificatio } \\
\text { n- } \\
\text { evaporation }\end{array}$ & $\begin{array}{l}\text { solid lipid } \\
\text { nanoparticl } \\
\text { e }\end{array}$ & $\begin{array}{l}\text { glyceryl behenate, } \\
\text { glyceryl monostearate, } \\
\text { sodium alginate }\end{array}$ & in & $\begin{array}{l}\text { plasma } \\
\text { insulin level }\end{array}$ & $\begin{array}{l}\text { RQ1, } \\
\text { RQ2, } \\
\text { RQ3, RQ4 }\end{array}$ \\
\hline $\begin{array}{l}6 \\
5\end{array}$ & $\begin{array}{l}\text { Heade } \\
\text { et al }\end{array}$ & $\begin{array}{l}20 \\
21\end{array}$ & MDPI & $\begin{array}{l}\text { Pharmaceutic } \\
\mathrm{s}(6.321)\end{array}$ & $\mathrm{N} / \mathrm{A}$ & $\begin{array}{l}\text { nanoparticl } \\
\text { e }\end{array}$ & BiPro WPI (97\%) & $\begin{array}{l}\text { in } \\
\text { vivo }\end{array}$ & \begin{tabular}{|l} 
plasma \\
insulin level
\end{tabular} & RQ3, RQ4 \\
\hline 6 & Agraw & 20 & $\begin{array}{l}\text { Royal } \\
\text { Society of }\end{array}$ & RSC Advances & $\mathrm{N} / \mathrm{A}$ & nanoparticl & $\begin{array}{l}\text { chitosan, poly(sodium } \\
\text { 4-styrenesulfonate) }\end{array}$ & in & plasma & RQ3, RQ4 \\
\hline
\end{tabular}




\begin{tabular}{|c|c|c|c|c|c|c|c|c|c|c|}
\hline $\begin{array}{l}\mathrm{N} \\
\mathrm{o}\end{array}$ & Author & $\begin{array}{l}\text { Yea } \\
\mathrm{r}\end{array}$ & Publisher & $\begin{array}{l}\text { Journal and } \\
\text { Impact Factor }\end{array}$ & Method & $\begin{array}{l}\text { Delivery } \\
\text { System }\end{array}$ & Formulation & $\begin{array}{l}\text { Test } \\
\text { Meth } \\
\text { od }\end{array}$ & $\begin{array}{l}\text { BA } \\
\text { Parameters }\end{array}$ & $\begin{array}{l}\text { Theme } \\
\text { Classifica } \\
\text { tion }\end{array}$ \\
\hline 6 & al et al & 15 & Chemistry & $(3.361)$ & & $\mathrm{e}$ & (PSS) & vivo & insulin level & \\
\hline $\begin{array}{l}6 \\
7\end{array}$ & Xu et al & $\begin{array}{l}20 \\
17\end{array}$ & Elsevier & \begin{tabular}{|l|} 
Materials \\
Science and \\
Engineering C \\
$(7.328)$
\end{tabular} & $\mathrm{N} / \mathrm{A}$ & liposome & $\begin{array}{l}\text { PLGA, asam folat, } \\
\text { kitosan, PVA }\end{array}$ & in & $\begin{array}{l}\text { plasma } \\
\text { insulin level }\end{array}$ & RQ3, RQ4 \\
\hline $\begin{array}{l}6 \\
8\end{array}$ & $\begin{array}{l}\text { Zhu et } \\
\text { al }\end{array}$ & $\begin{array}{l}20 \\
16\end{array}$ & $\begin{array}{l}\text { Taylor \& } \\
\text { Francis }\end{array}$ & $\begin{array}{l}\text { Drug Delivery } \\
(3.095)\end{array}$ & $\mathrm{N} / \mathrm{A}$ & $\begin{array}{l}\text { nanoparticl } \\
\mathrm{e}\end{array}$ & $\begin{array}{l}\text { PLGA, cell-penetrating } \\
\text { peptides (R8, Tat, } \\
\text { penetratin), PVA }\end{array}$ & $\begin{array}{l}\text { in } \\
\text { vivo }\end{array}$ & $\begin{array}{l}\text { plasma } \\
\text { insulin level }\end{array}$ & RQ3, RQ4 \\
\hline $\begin{array}{l}6 \\
9\end{array}$ & $\begin{array}{l}\text { Liu et } \\
\text { al }\end{array}$ & $\begin{array}{l}20 \\
16\end{array}$ & $\begin{array}{l}\text { Taylor \& } \\
\text { Francis }\end{array}$ & $\begin{array}{l}\text { Drug Delivery } \\
(3.095)\end{array}$ & $\mathrm{N} / \mathrm{A}$ & nanoparticl & $\begin{array}{l}\text { PLGA-mPEG } \\
\text { copolymers, Chitosan }\end{array}$ & in & $\begin{array}{l}\text { plasma } \\
\text { insulin level }\end{array}$ & $\begin{array}{l}\text { RQ1, } \\
\text { RQ2, } \\
\text { RQ3, RQ4 }\end{array}$ \\
\hline $\begin{array}{l}7 \\
0\end{array}$ & $\begin{array}{l}\text { Kaur et } \\
\text { al }\end{array}$ & $\begin{array}{l}20 \\
21\end{array}$ & Elsevier & \begin{tabular}{|l|} 
International \\
Journal of \\
Biological \\
Macromolecul \\
es (6.953)
\end{tabular} & $\mathrm{N} / \mathrm{A}$ & emulsion & piperin, albumin & $\begin{array}{l}\text { in } \\
\text { vivo }\end{array}$ & $\begin{array}{l}\text { plasma } \\
\text { insulin level }\end{array}$ & RQ3, RQ4 \\
\hline $\begin{array}{l}7 \\
1\end{array}$ & $\begin{array}{l}\text { Zhang } \\
\text { et al }\end{array}$ & $\begin{array}{l}20 \\
15\end{array}$ & Elsevier & \begin{tabular}{|l|} 
Indian \\
Journal of \\
Pharmaceutic \\
al Education \\
and Research \\
$(0.425)$
\end{tabular} & $\mathrm{N} / \mathrm{A}$ & nanoparticl & $\begin{array}{l}\text { PGA-g-DA, DMSO, } \\
\text { Trimethyl Chitosan } \\
\text { TMC/TMC-CSK, } \\
\text { tripolyphosphate } \\
\text { (TPP), magnesium } \\
\text { sulphate (MgSO4) }\end{array}$ & $\begin{array}{l}\text { in } \\
\text { vivo }\end{array}$ & $\begin{array}{l}\text { glucose } \\
\text { insulin level }\end{array}$ & $\begin{array}{l}\text { RQ1, } \\
\text { RQ2, } \\
\text { RQ3, RQ4 }\end{array}$ \\
\hline $\begin{array}{l}7 \\
2\end{array}$ & $\begin{array}{l}\text { Wang } \\
\text { et al }\end{array}$ & $\begin{array}{l}20 \\
20\end{array}$ & Springer & \begin{tabular}{|l|} 
Journal of \\
Nanobiotechn \\
ology \\
$(10.435)$
\end{tabular} & $\mathrm{N} / \mathrm{A}$ & $\begin{array}{l}\text { lipid } \\
\text { nanoparticl } \\
\text { es }\end{array}$ & $\begin{array}{l}\text { Soya phosphatidyl } \\
\text { choline (LIPOID E80) }\end{array}$ & $\begin{array}{l}\text { in } \\
\text { vivo }\end{array}$ & $\begin{array}{l}\text { plasma } \\
\text { insulin level }\end{array}$ & $\begin{array}{l}\text { RQ1, } \\
\text { RQ2, } \\
\text { RQ3, RQ4 }\end{array}$ \\
\hline $\begin{array}{l}7 \\
3\end{array}$ & $\begin{array}{l}\text { Guha } \\
\text { et al }\end{array}$ & $\begin{array}{l}20 \\
16\end{array}$ & $\begin{array}{l}\text { Taylor \& } \\
\text { Francis }\end{array}$ & $\begin{array}{l}\text { Drug Delivery } \\
(3.095)\end{array}$ & $\begin{array}{l}\text { layer-by- } \\
\text { layer }\end{array}$ & $\begin{array}{l}\text { mesoporou } \\
\text { s silica } \\
\text { nanoparticl } \\
\text { es }\end{array}$ & $\begin{array}{l}\text { Tetra ethoxy silane } \\
\text { (TEOS), 1, 3, } 5 \text { tri } \\
\text { methyl benzene (TMB), } \\
\text { Pluronic P123, } \\
\text { Azodiisobutyronitrile, } \\
\text { PMV [poly (methacrylic } \\
\text { acidco-vinyl } \\
\text { triethoxylsilane)] }\end{array}$ & in & $\begin{array}{l}\text { plasma } \\
\text { insulin level }\end{array}$ & $\begin{array}{l}\text { RQ1, } \\
\text { RQ2, } \\
\text { RQ3, RQ4 }\end{array}$ \\
\hline $\begin{array}{l}7 \\
4\end{array}$ & $\begin{array}{l}\text { Ukai et } \\
\text { al }\end{array}$ & $\begin{array}{l}20 \\
20\end{array}$ & MDPI & $\begin{array}{l}\text { Pharmaceutic } \\
\text { s (6.321) }\end{array}$ & $\mathrm{N} / \mathrm{A}$ & $\begin{array}{l}\text { self- } \\
\text { emulsifying }\end{array}$ & $\begin{array}{l}\text { caprylic acid }(\geq 90 \%) \\
\text { capric acid }(\leq 3.0 \%), \\
\text { lauric acid }(\leq 3.0 \%), \\
\text { myristic acid }(\leq 3.0 \%) \text {, } \\
\text { palmitic acid }(\leq 1.0 \%)\end{array}$ & in & $\begin{array}{l}\text { glucose } \\
\text { insulin level }\end{array}$ & $\begin{array}{l}\text { RQ1, } \\
\text { RQ2, } \\
\text { RQ3, RQ4 }\end{array}$ \\
\hline $\begin{array}{l}7 \\
5\end{array}$ & $\begin{array}{l}\text { Boushr } \\
\text { a et al }\end{array}$ & $\begin{array}{l}20 \\
19\end{array}$ & Elsevier & \begin{tabular}{|l|} 
Journal of \\
Drug Delivery \\
Science and \\
Technology \\
$(3,981)$
\end{tabular} & $\begin{array}{l}\text { emulsificatio } \\
\text { n solvent- } \\
\text { evaporation }\end{array}$ & $\begin{array}{l}\text { Solid lipid } \\
\text { nanoparticl } \\
\text { es }\end{array}$ & PLGA, PEG & $\begin{array}{l}\text { in } \\
\text { vivo }\end{array}$ & $\begin{array}{l}\text { plasma } \\
\text { insulin level }\end{array}$ & $\begin{array}{l}\text { RQ1, } \\
\text { RQ2, } \\
\text { RQ3, RQ4 }\end{array}$ \\
\hline $\begin{array}{l}7 \\
6\end{array}$ & $\begin{array}{l}\text { Elkhati } \\
\text { b et al }\end{array}$ & $\begin{array}{l}20 \\
21\end{array}$ & $\begin{array}{l}\text { Pharmace } \\
\text { utical } \\
\text { Society of } \\
\text { Japan }\end{array}$ & $\begin{array}{l}\text { Biological and } \\
\text { Pharmaceutic } \\
\text { al Bulletin } \\
(2.233)\end{array}$ & $\begin{array}{l}\text { ionotropic } \\
\text { pregelation }\end{array}$ & $\begin{array}{l}\text { Nanoparticl } \\
\text { e }\end{array}$ & $\begin{array}{l}\text { sodium alginate, } \\
\text { chitosan, dextran } \\
\text { sulphate, calcium } \\
\text { chloride dehydrate }\end{array}$ & $\begin{array}{l}\text { in } \\
\text { vivo }\end{array}$ & $\begin{array}{l}\text { plasma } \\
\text { insulin level }\end{array}$ & $\begin{array}{l}\text { RQ1, } \\
\text { RQ2, } \\
\text { RQ3, RQ4 }\end{array}$ \\
\hline
\end{tabular}

\section{CONCLUSION}

Oral insulin faces various challenges in the gastrointestinal tract, such as insulin degradation by proteolytic enzymes at acidic gastric $\mathrm{pH}$ to lack of insulin permeability in intestinal epithelial cells. Various manufacturing techniques must be adapted to the physicochemical properties of insulin to maintain insulin stability so that it can provide an optimal therapeutic effect. The materials used in the manufacture of nanocarriers are very influential in increasing the bioavailability of oral insulin due to their effect in paving the way for insulin across various barriers in the digestive tract. Until now, from various studies of oral insulin that have been developed, it has succeeded in obtaining oral insulin bioavailability of $73.10 \%$ achieved by using a mesoporous silica nanoparticles (MSN) delivery system with a layer-bylayer technique coated with a polymer [poly (methacrylic acid-co-vinyl triethoxylsilane)] (PMV)]. 


\section{SUGGESTIONS}

Based on the results of this systematic review, it can then be used as a basis for the development of new oral insulin formulas for bioavailability testing through in vivo studies. Future studies are expected to overcome the three main barriers to oral insulin while having a greater insulin loading capacity to achieve higher bioavailability.

\section{ACKNOWLEDGEMENT}

The author would like to thank the Ministry of Education and Culture of the Republic of Indonesia for the funds provided in the 2021 Student Creativity Program activities and Brawijaya University for the facilities provided.

\section{REFERENCES}

1. WHO; Diabetes, 2021. Available from: https://www.who.int/news-room/fact-sheets/detail/diabetes

2. Saeedi P, Petersohn I, Salpea P, Malanda B, Karuranga S, Unwin $\mathrm{N}$, et al. "Global and regional diabetes prevalence estimates for 2019 and projections for 2030 and 2045: Results from the International Diabetes Federation Diabetes Atlas, 9(th) edition". Diabetes Res Clin Pract, 2019; 157:107843. https://doi.org/10.1016/j.diabres.2019.107843

2 (a). Kementerian Kesehatan RI. CEGAH, CEGAH, DAN CEGAH: SUARA DUNIA PERANGI DIABETES. Indonesia; 2018. Available at:

https://www.kemkes.go.id/article/print/18121200001/cegahcegah-dan-cegah-suara-dunia-perangi-diabetes.html. Accessed February 8, 2021.

3. Shah RB, Patel M, Maahs DM, Shah VN, "Insulin delivery methods: Past, present and future" Int J Pharm Investig, 2016; 6(1):1-9. Available from: https://pubmed.ncbi.nlm.nih.gov/27014614 https://doi.org/10.4103/2230-973X.176456

4. Freeland B, Farber MS. "A Review of Insulin for the Treatment of Diabetes Mellitus" Home Healthc now, 2016 Sep; 34(8):416-23. https://doi.org/10.1097/NHH.0000000000000446

5. Wong CY, Martinez J, Dass CR, "Oral delivery of insulin for treatment of diabetes: status quo, challenges and opportunities" J Pharm Pharmacol, 2016 Sep; 68(9):1093-108. Available from: https://doi.org/10.1111/jphp.12607

6. Krishnaiah Y. "Pharmaceutical Technologies for Enhancing Oral Bioavailability of Poorly Soluble Drugs" J Bioequiv Availab, 2010 Jan; 1(2). https://doi.org/10.4172/jbb.1000027

7. Bhosle VK, Altit G, Autmizguine J, Chemtob S, "18 - Basic Pharmacologic Principles. In: Polin RA, Abman SH, Rowitch DH, Benitz WE, Fox WWBT-F and NP (Fifth E, editors" Elsevier, 2017; 187-201. DOI: https://www.sciencedirect.com/science/article/pii/B9780323 352147000184

8. Savjani KT, Gajjar AK, Savjani JK, "Drug Solubility: Importance and Enhancement Techniques" Aktay G, Du Y-Z, Torrado J, editors, ISRN Pharm [Internet], 2012; 2012:195727. DOI: https://doi.org/10.5402/2012/195727

9. Fonte P, Araújo F, Reis S, Sarmento B, "Oral insulin delivery: How far are we?" J Diabetes Sci Technol, 2013; 7(2):520-31. https://doi.org/10.1177/193229681300700228

10. Singh AP, Guo Y, Singh A, Xie W, Jiang P, "Developments in encapsulation of insulin: Is oral delivery now possible?" J Pharm Biopharm Res, 2019; 1(2):74-93. https://doi.org/10.25082/JPBR.2019.02.005

11. Wong CY, Al-Salami H, Dass CR, "Fabrication techniques for the preparation of orally administered insulin nanoparticles" J Drug Target, 2021 Apr 21; 29(4):365-86. DOI: https://doi.org/10.1080/1061186X.2020.1817042

12. Rowley J, Slack F, "Conducting a literature review" Manag Res News, 2004; 27(6):31-9.

\section{https://doi.org/10.1108/01409170410784185}

13. Page M, McKenzie J, Bossuyt P, Boutron I, Hoffmann T, Mulrow C, "The PRISMA 2020 statement: an updated guideline for reporting systematic reviews" BMJ, 2021; 372(71). https://doi.org/10.1136/bmj.n71

14. Han X, Lu Y, Xie J, Zhang E, Zhu H, Du H, et al, "Zwitterionic micelles efficiently deliver oral insulin without opening tight junctions" Nat Nanotechnol, 2020; 15(7):605-14. DOI: http://dx.doi.org/10.1038/s41565-020-0693-6

15. Jaafar MHM, Hamid KA, "Chitosan-Coated Alginate Nanoparticles Enhanced Absorption Profile of Insulin Via Oral Administration" Curr Drug Deliv, 2019; 16(7):672-86. https://doi.org/10.2174/1567201816666190620110748

16. Iqbal M, Zafar N, Fessi H, Elaissari A, "Double emulsion solvent evaporation techniques used for drug encapsulation" Int J Pharm, 2015 Dec; 496(2):173-90. https://doi.org/10.1016/j.ijpharm.2015.10.057

17. Chen T, Li S, Zhu W, Liang Z, Zeng Q, "Self-assembly pH-sensitive chitosan/alginate coated polyelectrolyte complexes for oral delivery of insulin" J Microencapsul [Internet], 2019; 36(1):96107. DOI: https://doi.org/10.1080/02652048.2019.1604846

18. Liu L, Zhou C, Xia X, Liu Y, "Self-assembled lecithin/chitosan nanoparticles for oral insulin delivery: Preparation and functional evaluation" Int J Nanomedicine, 2016; 11:761-9. https://doi.org/10.2147/IJN.S96146

19. Guha A, Biswas N, Bhattacharjee K, Sahoo N, Kuotsu K, "pH responsive cylindrical MSN for oral delivery of insulin-design, fabrication and evaluation" Drug Deliv, 2016; 23(9):3552-61. https://doi.org/10.1016/j.biomaterials.2017.03.028

20. He Z, Santos JL, Tian H, Huang H, Hu Y, Liu L, et al, "Scalable fabrication of size-controlled chitosan nanoparticles for oral delivery of insulin" Biomaterials, 2017; 130:28-41. DOI: http://dx.doi.org/10.1016/j.biomaterials.2017.03.028

21. Malathi S, Nandhakumar $P$, Pandiyan $V$, Webster TJ Balasubramanian S, "Novel PLGA-based nanoparticles for the oral delivery of insulin" Int J Nanomedicine, 2015; 10:2207-18. https://doi.org/10.2147/IJN.S67947

22. Wu S, Bin W, Tu B, Li X, Wang W, Liao S, et al, "A Delivery System for Oral Administration of Proteins/Peptides Through Bile Acid Transport Channels" J Pharm Sci, 2019; 108(6):2143-52. DOI: https://doi.org/10.1016/j.xphs.2019.01.027

23. Liu M, Zhang J, Zhu X, Shan W, Li L, Zhong J, et al, "Efficient mucus permeation and tight junction opening by dissociable "mucus-inert" agent coated trimethyl chitosan nanoparticles for oral insulin delivery" J Control Release, 2016; 222:67-77. DOI: http://dx.doi.org/10.1016/j.jconrel.2015.12.008

24. Wu L, Liu M, Shan W, Zhu X, Li L, Zhang Z, et al, "Bioinspired butyrate-functionalized nanovehicles for targeted oral delivery of biomacromolecular drugs" J Control Release, 2017 262(July):273-83.

http://dx.doi.org/10.1016/j.jconrel.2017.07.045

25. Alsulays BB, Anwer MK, Soliman GA, Alshehri SM, Khafagy ES, "Impact of penetratin stereochemistry on the oral bioavailability of insulin-loaded solid lipid nanoparticles" Int J Nanomedicine, 2019; https://doi.org/10.2147/IJN.S225086

26. Wang A, Yang T, Fan W, Yang Y, Zhu Q Guo S, et al, "Protein Corona Liposomes Achieve Efficient Oral Insulin Delivery by Overcoming Mucus and Epithelial Barriers" Adv Healthc Mater, 2019; 8(12):1-11. https://doi.org/10.1002/adhm.201801123

27. Ji N, Hong Y, Gu Z, Cheng L, Li Z, Li C, "Chitosan coating of zeincarboxymethylated short-chain amylose nanocomposites improves oral bioavailability of insulin in vitro and in vivo" J Control Release, 2019; 313(July):1-13. https://doi.org/10.1016/j.jconrel.2019.10.006

28. Agrawal AK, Urimi D, Harde H, Kushwah V, Jain S, "Folate appended chitosan nanoparticles augment the stability, bioavailability and efficacy of insulin in diabetic rats following 
oral administration" RSC Adv, 2015; 5(127):105179-93. https://doi.org/10.1039/C5RA19115G

29. Fan W, Xia D, Zhu Q, Li X, He S, Zhu C, et al, "Functional nanoparticles exploit the bile acid pathway to overcome multiple barriers of the intestinal epithelium for oral insulin delivery" Biomaterials, 2018; 151:13-23. DOI: https://doi.org/10.1016/j.biomaterials.2017.10.022

30. Jafary Omid N, Bahari Javan N, Dehpour AR, Partoazar A, Rafiee Tehrani M, Dorkoosh F, "In-vitro and in-vivo cytotoxicity and efficacy evaluation of novel glycyl-glycine and alanyl-alanine conjugates of chitosan and trimethyl chitosan nano-particles as carriers for oral insulin delivery" Int J Pharm, 2018; 535(12):293-307. https://doi.org/10.1016/j.ijpharm.2017.11.020

31. Zhang L, Zhang YX, Qiu JN, Li J, Chen W, Guan YQ, "Preparation and Characterization of Hypoglycemic Nanoparticles for Oral Insulin Delivery" Biomacromolecules, 2017; 18(12):4281-91. https://doi.org/10.1021/acs.biomac.7b01322

32. Mumuni MA, Kenechukwu FC, Ofokansi KC, Attama AA, Díaz DD, "Insulin-loaded mucoadhesive nanoparticles based on mucinchitosan complexes for oral delivery and diabetes treatment" Carbohydr Polym, 2020; 229(June 2019):115506. DOI: https://doi.org/10.1016/j.carbpol.2019.115506

33. Ukai H, Iwasa K, Deguchi T, Morishita M, Katsumi H, Yamamoto A, "Enhanced intestinal absorption of insulin by capryol 90, a novel absorption enhancer in rats: Implications in oral insulin delivery" Pharmaceutics, 2020; 12(5):1-16 https://doi.org/10.3390/pharmaceutics12050462

34. Fang $Y$, Wang $Q$, Lin $X$, Jin $X$, Yang $D$, Gao S, et al, "Gastrointestinal Responsive Polymeric Nanoparticles for Oral Delivery of Insulin: Optimized Preparation, Characterization, and In Vivo Evaluation" J Pharm Sci, 2019; 108(9):2994-3002. DOI: https://doi.org/10.1016/j.xphs.2019.04.020

35. Zhang $\mathrm{P}, \mathrm{Xu} \mathrm{Y}$, Zhu $\mathrm{X}$, Huang $\mathrm{Y}$, "Goblet cell targeting nanoparticle containing drug-loaded micelle cores for oral delivery of insulin" Int J Pharm, 2015; 496(2):993-1005. https://doi.org/10.1016/j.ijpharm.2015.10.078

36. Sun L, Liu Z, Tian H, Le Z, Liu L, Leong KW, et al, "Scalable Manufacturing of Enteric Encapsulation Systems for SiteSpecific Oral Insulin Delivery" Biomacromolecules, 2019; 20(1):528-38. https://doi.org/10.1021/acs.biomac.8b01530

37. Li L, Jiang G, Yu W, Liu D, Chen H, Liu Y, et al, "Preparation of chitosan-based multifunctional nanocarriers overcoming multiple barriers for oral delivery of insulin" Mater Sci Eng C, 2017; 70(Part 2):278-86. DOI: http://dx.doi.org/10.1016/j.msec.2016.08.083

38. Liu C, Shan W, Liu M, Zhu X, Xu J, Xu Y, et al, "A novel ligand conjugated nanoparticles for oral insulin delivery" Drug Deliv, 2016; https://doi.org/10.3109/10717544.2015.1058433 23(6):2015-25

39. Sahoo P, Leong KH, Nyamathulla S, Onuki Y, Takayama K, Chung LY, "Chitosan complexed carboxymethylated iota-carrageenan oral insulin particles: Stability, permeability and in vivo evaluation" Mater Today Commun, 2019; 20(June):100557. DOI: https://doi.org/10.1016/j.mtcomm.2019.100557

40. Sheng J, He H, Han L, Qin J, Chen S, Ru G, et al, "Enhancing insulin oral absorption by using mucoadhesive nanoparticles loaded with LMWP-linked insulin conjugates" J Control Release, 2016; 233:181-90. https://doi.org/10.1016/j.jconrel.2016.05.015

41. Sun S, Liang N, Yamamoto H, Kawashima Y, Cui F, Yan P, "pHsensitive poly(lactide-co-glycolide) nanoparticle composite microcapsules for oral delivery of insulin" Int J Nanomedicine, 2015; 10:3489-98. https://doi.org/10.2147/IJN.S81715

42. Bravo-Alfaro DA, Muñoz-Correa MOF, Santos-Luna D, ToroVazquez JF, Cano-Sarmiento C, García-Varela R, et al, "Encapsulation of an insulin-modified phosphatidylcholine complex in a self-nanoemulsifying drug delivery system (SNEDDS) for oral insulin delivery" J Drug Deliv Sci Technol, 2020; 57(January):101622. DOI: https://doi.org/10.1016/j.jddst.2020.101622
43. Sheng J, Han L, Qin J, Ru G, Li R, Wu L, et al, "N -Trimethyl Chitosan Chloride-Coated PLGA Nanoparticles Overcoming Multiple Barriers to Oral Insulin Absorption" ACS Appl Mater Interfaces, 2015; 7(28):15430-41. https://doi.org/10.1021/acsami.5b03555

44. Wang X, Cheng D, Liu L, Li X, "Development of poly(hydroxyethyl methacrylate) nanogel for effective oral insulin delivery" Pharm Dev Technol, 2018; 23(4):351-7. DOI: https://doi.org/10.1080/10837450.2017.1295064

45. Wu J, Zheng Y, Liu M, Shan W, Zhang Z, Huang Y, "Biomimetic Viruslike and Charge Reversible Nanoparticles to Sequentially Overcome Mucus and Epithelial Barriers for Oral Insulin Delivery" ACS Appl Mater Interfaces, 2018; 10(12):9916-28. https://doi.org/10.1021/acsami.7b16524

46. Yazdi JR, Tafaghodi M, Sadri K, Mashreghi M, Nikpoor AR, Nikoofal-Sahlabadi S, et al, "Folate targeted PEGylated liposomes for the oral delivery of insulin: In vitro and in vivo studies" Colloids Surfaces B Biointerfaces, 2020; 194:111203. DOI: https://doi.org/10.1016/j.colsurfb.2020.111203

47. Hu X Bin, Tang TT, Li YJ, Wu JY, Wang JM, Liu XY, et al, "Phospholipid complex based nanoemulsion system for oral insulin delivery: Preparation, in vitro, and in vivo evaluations" Int J Nanomedicine, 2019; 14:3055-67. https://doi.org/10.2147/IJN.S198108

48. Liu C, Kou Y, Zhang X, Dong W, Cheng H, Mao S, "Enhanced oral insulin delivery via surface hydrophilic modification of chitosan copolymer based self-assembly polyelectrolyte nanocomplex" Int J Pharm, 2019; 554:36-47. DOI: https://doi.org/10.1016/j.ijpharm.2018.10.068

49. Liu J, Werner U, Funke M, Besenius M, Saaby L, Fanø M, et al, "SEDDS for intestinal absorption of insulin: Application of Caco2 and Caco-2/HT29 co-culture monolayers and intra-jejunal instillation in rats" Int J Pharm, 2019; 560:377-84. DOI: https://doi.org/10.1016/j.ijpharm.2019.02.014

50. Boushra M, Tous S, Fetih G, Xue HY, Wong HL, "Development of bi-polymer lipid hybrid nanocarrier (BLN) to improve the entrapment and stability of insulin for efficient oral delivery" J Drug Deliv Sci Technol, 2019; 49:632-41. DOI: https://doi.org/10.1016/j.jddst.2019.01.007

51. Shan W, Zhu X, Tao W, Cui Y, Liu M, Wu L, et al, "Enhanced Oral Delivery of Protein Drugs Using Zwitterion-Functionalized Nanoparticles to Overcome both the Diffusion and Absorption Barriers" ACS Appl Mater Interfaces, 2016; 8(38):25444-53.

52. Ji N, Hong Y, Gu Z, Cheng L, Li Z, Li C, "Binary and Tertiary Complex Based on Short-Chain Glucan and Proanthocyanidins for Oral Insulin Delivery" J Agric Food Chem, 2017; 65(40):8866-74. https://doi.org/10.1021/acs.jafc.7b03465

53. Xie S, Gong YC, Xiong XY, Li ZL, Luo YY, Li YP, "Targeted folateconjugated pluronic for the oral delivery of insulin" 2018.

54. Tian H, He Z, Sun C, Yang C, Zhao P, Liu L, et al, "Uniform CoreShell Nanoparticles with Thiolated Hyaluronic Acid Coating to Enhance Oral Delivery of Insulin" Adv Healthc Mater, 2018; 7(17):1-12. https://doi.org/10.1002/adhm.201800285

55. Guo F, Zhang M, Gao Y, Zhu S, Chen S, Liu W, et al, "Modified nanoparticles with cell-penetrating peptide and amphipathic chitosan derivative for enhanced oral colon absorption of insulin: preparation and evaluation" Drug Deliv, 2016; 23(6):2003-14.

https://doi.org/10.3109/10717544.2015.1048489

56. Zhang Y, Xiong GM, Ali Y, Boehm BO, Huang YY, Venkatraman S, "Layer-by-layer coated nanoliposomes for oral delivery of

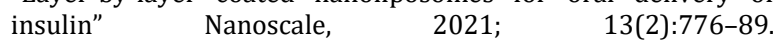
https://doi.org/10.1039/D0NR06104B

57. Alibolandi M, Alabdollah F, Sadeghi F, Mohammadi M, Abnous K, Ramezani M, et al, "Dextran-b-poly (lactide-co-glycolide) polymersome for oral delivery of insulin: In vitro and in vivo evaluation" J Control Release, 2016; 227:58-70. DOI: http://dx.doi.org/10.1016/j.jconrel.2016.02.031

58. Kim KS, Kwag DS, Hwang HS, Lee ES, Bae YH, “Immense Insulin 
Intestinal Uptake and Lymphatic Transport using Bile Acid Conjugated Partially Uncapped Liposome" Mol Pharm, 2018; 176(1):139-48.

59. Zhang L, Qin H, Li J, Qiu JN, Huang JM, Li MC, et al, "Preparation and characterization of layer-by-layer hypoglycemic nanoparticles with pH-sensitivity for oral insulin delivery" J Mater Chem B, 2018; 6(45):7451-61. https://doi.org/10.1039/С8ТВ02113A

60. Verma A, Sharma S, Gupta PK, Singh A, Teja BV, Dwivedi P, et al, "Vitamin B12 functionalized layer by layer calcium phosphate nanoparticles: A mucoadhesive and $\mathrm{pH}$ responsive carrier for improved oral delivery of insulin" Acta Biomater, 2016; 31:288300. DOI: http://dx.doi.org/10.1016/j.actbio.2015.12.017

61. Fukuoka Y, Khafagy ES, Goto T, Kamei N, Takayama K, Peppas NA, et al, "Combination strategy with complexation hydrogels and cell-penetrating peptides for oral delivery of insulin" Biol Pharm Bull, 2018; 41(5):811-4 https://doi.org/10.1248/bpb.b17-00951

62. Yan C, Gu J, Lv Y, Shi W, Huang Z, Liao Y, “ $5 \beta$-Cholanic Acid/Glycol Chitosan Self-Assembled Nanoparticles CHA/GC-NPs) for Enhancing the Absorption of FDs and Insulin by Rat Intestinal Membranes" AAPS PharmSciTech, 2019; 20(1):1-8. https://doi.org/10.1208/s12249-018-1242-6

63. Wang J, Kong M, Zhou Z, Yan D, Yu X, Cheng X, et al, "Mechanism of surface charge triggered intestinal epithelial tight junction opening upon chitosan nanoparticles for insulin oral delivery" Carbohydr Polym, 2017;157:596-602. DOI: http://dx.doi.org/10.1016/j.carbpol.2016.10.021

64. Chen X, Ren Y, Feng Y, Xu X, Tan H, Li J, “Cp1-11 peptide/insulin complex loaded $\mathrm{pH}$-responsive nanoparticles with enhanced oral bioactivity" Int J Pharm, 2019; 562:23-30. DOI: https://doi.org/10.1016/j.ijpharm.2019.03.020

65. Bahman F, Taurin S, Altayeb D, Taha S, Bakhiet M, Greish K, "Oral insulin delivery using poly (Styrene co-Maleic acid) micelles in a diabetic mouse model" Pharmaceutics, 2020; 12(11):1-17.

https://doi.org/10.3390/pharmaceutics12111026

66. Shrestha N, Araújo F, Shahbazi MA, Mäkilä E, Gomes MJ, Herranz-Blanco B, et al, "Thiolation and Cell-Penetrating Peptide Surface Functionalization of Porous Silicon Nanoparticles for Oral Delivery of Insulin" Adv Funct Mater, 2016; https://doi.org/10.1002/adfm.201505252

67. Zhou X, Wu H, Long R, Wang S, Huang H, Xia Y, et al, "Oral delivery of insulin with intelligent glucose-responsive switch for blood glucose regulation" J Nanobiotechnology, 2020; 18(1):116. DOI: https://doi.org/10.1186/s12951-020-00652-z

68. Winarti L, Suwaldi, Martien R, Hakim L, "Formulation of insulin self nanoemulsifying drug delivery system and its in vitro-in vivo study" Indones J Pharm, 2018; 29(3):157-66. https://doi.org/10.14499/indonesianjpharm29iss3pp157

69. Sun S, Liang N, Gong X, An W, Kawashima Y, Cui F, et al, "Multifunctional composite microcapsules for oral delivery of insulin" Int J Mol Sci, 2017; 18(1). https://doi.org/10.3390/ijms18010054

70. Zhang $\mathrm{Y}$, Xiong $\mathrm{M}$, Ni X, Wang J, Rong H, Su Y, et al, "VirusMimicking Mesoporous Silica Nanoparticles with an Electrically Neutral and Hydrophilic Surface to Improve the Oral Absorption of Insulin by Breaking through Dual Barriers of the Mucus Layer and the Intestinal Epithelium" ACS Appl Mater Interfaces, 2021; 13(15):18077-88. https://doi.org/10.1021/acsami.1c00580

71. Zeng Z, Dong C, Zhao P, Liu Z, Liu L, Mao HQ, et al, "Scalable Production of Therapeutic Protein Nanoparticles Using Flash Nanoprecipitation" Adv Healthc Mater, 2018; 8(6):1-7. https://doi.org/10.1002/adhm.201801010

72. He H, Wang P, Cai C, Yang R, Tang X, "VB12-coated Gel-Core-SLN containing insulin: Another way to improve oral absorption" Int J Pharm, 2015; 493(1-2):451-9. DOI: http://dx.doi.org/10.1016/j.ijpharm.2015.08.004
73. Ansari MJ, Anwer MK, Jamil S, Al-Shdefat R, Ali BE, Ahmad MM, et al, "Enhanced oral bioavailability of insulin-loaded solid lipid nanoparticles: pharmacokinetic bioavailability of insulin-loaded solid lipid nanoparticles in diabetic rats" Drug Deliv, 2016; 23(6):1972-9.

https://doi.org/10.3109/10717544.2015.1039666

74. Niu Z, Tedesco E, Benetti F, Mabondzo A, Montagner IM, Marigo $\mathrm{I}$, et al, "Rational design of polyarginine nanocapsules intended to help peptides overcoming intestinal barriers" J Control Release, 2017; 263:4-17. https://doi.org/10.1016/j.jconrel.2017.02.024

75. Boushra M, Tous S, Fetih G, Korzekwa K, Lebo DB, Xue HY, et al, "Development and evaluation of viscosity-enhanced nanocarrier (VEN) for oral insulin delivery" Int J Pharm, 2016; 511(1):46272. DOI: http://dx.doi.org/10.1016/j.ijpharm.2016.07.016

76. Koland M, Anchan RB, Mukund SG, Sindhoor SM, "Design and investigation of alginate coated solid lipid nanoparticles for oral insulin delivery" Indian J Pharm Educ Res, 2021; 55(2):383-94. https://doi.org/10.5530/ijper.55.2.76

77. Deng W, Xie Q, Wang H, Ma Z, Wu B, Zhang X, "Selenium nanoparticles as versatile carriers for oral delivery of insulin: Insight into the synergic antidiabetic effect and mechanism" Nanomedicine Nanotechnology, Biol Med, 2017; 13(6):1965-74 DOI: http://dx.doi.org/10.1016/j.nano.2017.05.002

78. Singh S, Kushwah V, Agrawal AK, Jain S, "Insulin- and quercetinloaded liquid crystalline nanoparticles: Implications on oral bioavailability, antidiabetic and antioxidant efficacy" Nanomedicine, 2018; 13(5):521-37. https://doi.org/10.2217/nnm-2017-0278

79. Heade J, McCartney F, Chenlo M, Marro OM, Severic M, Kent R, et al, "Synthesis and in vivo evaluation of insulin-loaded whey beads as an oral peptide delivery system" Pharmaceutics, 2021; 13(5):1-18. https://doi.org/10.3390/pharmaceutics13050656

80. Chen S, Guo F, Deng T, Zhu S, Liu W, Zhong H, et al, "Eudragit S100-Coated Chitosan Nanoparticles Co-loading Tat for Enhanced Oral Colon Absorption of Insulin" AAPS PharmSciTech, 2017; 18(4):1277-87. https://doi.org/10.1208/s12249-016-0594-z

81. Agrawal AK, Kumar K, Swarnakar NK, Kushwah V, Jain S, "liquid Crystalline Nanoparticles": Rationally Designed Vehicle to Improve Stability and Therapeutic Efficacy of Insulin Following Oral Administration" Mol Pharm, 2017; 14(6):1874-82. https://doi.org/10.1021/acs.molpharmaceut.6b01099

82. Kaur I, Nallamothu B, Kuche K, Katiyar SS, Chaudhari D, Jain S, "Exploring protein stabilized multiple emulsion with permeation enhancer for oral delivery of insulin" Int J Biol Macromol, 2021; 167:491-501. DOI: https://doi.org/10.1016/j.ijbiomac.2020.11.190

83. Zhu S, Chen S, Gao Y, Guo F, Li F, Xie B, et al, "Enhanced oral bioavailability of insulin using PLGA nanoparticles co-modified with cell-penetrating peptides and Engrailed secretion peptide (Sec)" Drug Deliv, 2016; 23(6):1980-91. https://doi.org/10.3109/10717544.2015.1043472

84. Urimi D, Agrawal AK, Kushwah V, Jain S, "Polyglutamic Acid Functionalization of Chitosan Nanoparticles Enhances the Therapeutic Efficacy of Insulin Following Oral Administration" AAPS PharmSciTech, 2019; 20(3):1-14. https://doi.org/10.1208/s12249-019-1330-2

85. Wang T, Shen L, Zhang Y, Li H, Wang Y, Quan D, "Oil-soluble" reversed lipid nanoparticles for oral insulin delivery" J Nanobiotechnology, 2020; https://doi.org/10.1186/s12951-020-00657-8

86. Xu B, Jiang G, Yu W, Liu D, Liu Y, Kong X, et al, "Preparation of poly(lactic-co-glycolic acid) and chitosan composite nanocarriers via electrostatic self assembly for oral delivery of insulin" Mater Sci Eng C, 2017;78:420-8. DOI: http://dx.doi.org/10.1016/j.msec.2017.04.113

87. El Leithy ES, Abdel-Bar HM, Ali RAM, "Folate-chitosan nanoparticles triggered insulin cellular uptake and improved in 
vivo hypoglycemic activity" Int J Pharm, 2019; 571:118708. DOI: https://doi.org/10.1016/j.ijpharm.2019.118708

88. Elkhatib MM, Ali AI, Al-Badrawy AS, "In vitro and in vivo comparative study of oral nanoparticles and gut iontophoresis as oral delivery systems for insulin" Biol Pharm Bull, 2021; 44(2):251-8. https://doi.org/10.1248/bpb.b20-00737

89. Elsayed AM, Khaled AH, Al Remawi MM, Qinna NA, Farsakh HA, Badwan AA, "Low molecular weight chitosan-insulin complexes solubilized in a mixture of self-assembled labrosol and plurol oleaque and their glucose reduction activity in rats" Mar Drugs, 2018; 16(1). https://doi.org/10.3390/md16010032

90. Zheng Y, Wu J, Shan W, Wu L, Zhou R, Liu M, et al, "Multifunctional Nanoparticles Enable Efficient Oral Delivery of Biomacromolecules via Improving Payload Stability and Regulating the Transcytosis Pathway" ACS Appl Mater Interfaces, $\quad 2018$; $10(40): 34039-49$. https://doi.org/10.1021/acsami.8b13707 